\title{
Statistical Analysis of Metabolic Pathways of Brain Metabolism at Steady State
}

\author{
R. Occhipinti, ${ }^{1}$ M. A. Puchowicz, ${ }^{2}$ J. C. LaManna, ${ }^{2}$ E. Somersalo, ${ }^{3}$ and D. Calvetti ${ }^{1}$ \\ ${ }^{1}$ Department of Mathematics and Center for Modeling Integrated Metabolic Systems, Case Western Reserve University, \\ 10900 Euclid Avenue, Cleveland, OH 44106, USA; ${ }^{2}$ Department of Anatomy and Center for Modeling Integrated Metabolic \\ Systems, Case Western Reserve University, 10900 Euclid Avenue, Cleveland, OH 44106, USA; and ${ }^{3}$ Institute of Mathematics, \\ Helsinki University of Technology, P.O. Box 1100 FIN-02015Hut, Finland
}

(Received 6 October 2006; accepted 25 January 2007; published online 24 March 2007)

\begin{abstract}
The estimation of metabolic fluxes for brain metabolism is important, among other things, to test the validity of different hypotheses which have been proposed in the literature. The metabolic model that we propose considers, in addition to the blood compartment, the cytosol, and mitochondria of both astrocyte and neuron, including detailed metabolic pathways. In this work we use a recently developed methodology to perform a statistical Flux Balance Analysis (FBA) for this model. The methodology recasts the problem in the form of Bayesian statistical inference and therefore can take advantage of qualitative information about brain metabolism for the simultaneous estimation of all reaction fluxes and transport rates at steady state. By a Markov Chain Monte Carlo (MCMC) sampling method, we are able to provide for each reaction flux and transport rate a distribution of possible values. The analysis of the histograms of the reaction fluxes and transport rates provides a very useful tool for assessing the validity of different hypotheses about brain energetics proposed in the literature, and facilitates the design of the pathways network that is in accordance with what is understood of the functioning of the brain. In this work, we focus on the analysis of biochemical pathways within each cell type (astrocyte and neuron) at different levels of neural activity, and we demonstrate how statistical tools can help implement various bounds suggested by experimental data.
\end{abstract}

Keywords-Flux balance, Brain, Markov Chain Monte Carlo, Bayesian statistics, Multicompartment model, Metabolism, Neural activity.

\section{INTRODUCTION}

Despite many hypotheses about the relation between neural activity and energy metabolism in brain tissue, until now a detailed mathematical model

Address correspondence to D. Calvetti, Department of Mathematics and Center for Modeling Integrated Metabolic Systems, Case Western Reserve University, 10900 Euclid Avenue, Cleveland, OH 44106, USA. Electronic mail: daniela.calvetti@case.edu comprising simultaneously various of the components which might contribute to fuel generation remains to be discussed. A model which considers several key important metabolic reactions related to energy metabolism in astrocyte and neuron together with pertinent inter and intracellular transporter systems is the first step towards being able to test in silico the implications of the various theories and hypotheses about brain metabolism.

The estimation of reaction fluxes and transmembrane transport rates in and across different compartments at steady state is usually referred to as Flux Balance Analysis (FBA) in the literature. FBA is a very useful tool to verify the hypotheses on the role of different metabolic pathways related to brain function that have been proposed. Since direct measurements of several of the reaction fluxes and transport rates are not available, it is quite helpful to be able to estimate them in silico. Furthermore, by performing FBA we can predict the activation level of the various pathways at different neural activation intensity according to the various hypotheses proposed. This information can then serve as a guideline for acquiring measured data of key quantities.

The fuel utilization in brain metabolism has been the topic of extensive research and has given rise to a number of different hypotheses, which are difficult to verify experimentally because of the inherent obstacles in obtaining in vivo measurements of the pertinent quantities. ${ }^{4,17,19,24}$ As experiments have suggested that the fueling of the brain follows various metabolic pathways depending on the level of neural activity, a suitable model able to detect the shift in metabolic pathways will necessarily be quite complex. ${ }^{7} \mathrm{We}$ compare different hypotheses using a mathematical model which includes blood, astrocyte, and neuron compartments, and where each cell type is further subdivided into cytosol and mitochondria. We remark 
that this is not the only possible compartmentalization in the study of cellular brain metabolism. The resulting five-compartment model of brain tissue consists of reactions and transports of metabolites and metabolic intermediates which need to be considered simultaneously to address the complexity of the system. Our model synthesizes several components that have been investigated individually in the literature. In fact, although several studies have focused on glycolysis in the astrocyte, on the neurotransmitter glutamate-glutamine cycle, or on the astrocyte-neuron lactate shuttle (ANLS), a mathematical model which can be used to better understand the interplay between them has not been previously proposed in the literature.

The increasing importance of computational methods to study complex systems has been recently pointed out in the context of the shift of systems biology from the classical reductionist to an inclusive approach, wherein instead of simplifying the model to a mathematically tractable one, a detailed - and therefore quite complex-model is considered and the interactions between different components are investigated. ${ }^{11}$ By being able to estimate all reaction fluxes and transport rates at once, we can in fact design in silico experiments which are impossible or unethical to perform in vivo to help further explain the different hypotheses about brain metabolism proposed in the literature. Although the recent increase in computational power is certainly helpful when running simulations of complex systems, it does not remove the challenge of identifying complex systems even in the presence of abundant data. This has been the main motivation for designing approaches which integrate various types of constraints, including bounds, stoichiometry and uncertainty both in the data and in the model. The need for sophisticated methodologies has become particularly clear when dealing with high throughput networks and systems which arise in gene expression research.

The FBA estimation problem for detailed models of complex systems is underdetermined, because the number of unknowns exceeds the available data and conditions that have to be satisfied for the system to be at steady state. The constraint of the stoichiometry on the reaction fluxes can be used to compensate in part for the insufficient data. In addition, some of the quantities of interest are often believed to take on values in certain intervals, as suggested, e.g., by experimental evidence or biochemical and physiological considerations. These stoichiometric and bound constraints for FBA have been used in the context of a Linear Programming (LP) environment, together with the introduction of a suitable objective function, to identify a feasible space of reaction fluxes.,11,21,26 Since the feasible solution space is, in general, quite large, additional tools, e.g., the extreme pathways methodology, have been designed to tighten the range of possible values. ${ }^{22,23}$

In this work we propose a new five-compartment model of brain metabolism, which considers 75 reactions and 34 transports across membranes between compartments, and we apply a new methodology for FBA to estimate reaction fluxes, transport rates and concentrations of biochemical species in the venous blood at basal steady state at different levels of neural activity (Fig. 1). This model includes several metabolic intermediates that, although not relevant for the study of the steady state case, can play an important role in the investigation of the dynamic response of the system. There are several reasons why these intermediates should be included in a dynamic model. Some of them can in fact be measured and therefore used to identify and validate the model. Furthermore, some of the intermediate reactions might depend on enzymes that play a key role to understand pathological conditions. Note that since the proposed model results in not spatially distributed, it lumps glutamatergic and GABAergic neurons into one compartment.

Our analysis follows a new paradigm, based on the Bayesian statistical framework, first introduced for investigating skeletal muscle metabolism using a twocompartment model. ${ }^{3,4}$ This Bayesian framework is naturally suited for the inclusion of various types of information about the system described by the model, including qualitative information which are difficult to use, for example, in the LP framework. ${ }^{4}$ In this approach, the FBA becomes a statistical inference problem, and therefore its solution consists of statistical distributions of the reaction fluxes and transport rates. While the mean of these distributions can be used as estimates, the actual shape of the densities indicates how the value might fluctuate over the population represented by our model. The exploration of the probability densities is done by Markov Chain Monte Carlo (MCMC) techniques.

We focus on the identification and analysis of preferred metabolic pathways in the cell metabolism under different levels of energy demand as a function of neural activity. In particular, we seek to identify the neural energy shuttling of carbon units through metabolic pathways, such as glycolysis, TCA cycle, glutamate/glutamine shuttle, also referred to as the Vcycle, astrocyte-neuron lactate shuttle, GABA shunt (Fig. 1). In particular, we are interested in the proportions of the metabolic fuel supplied via neuronal glycolysis, lactate shuttle from the astrocyte and via glutamate supply through the V-cycle as a function of neural activity intensity. One of the main points of this article is also to demonstrate the flexibility of the sta- 


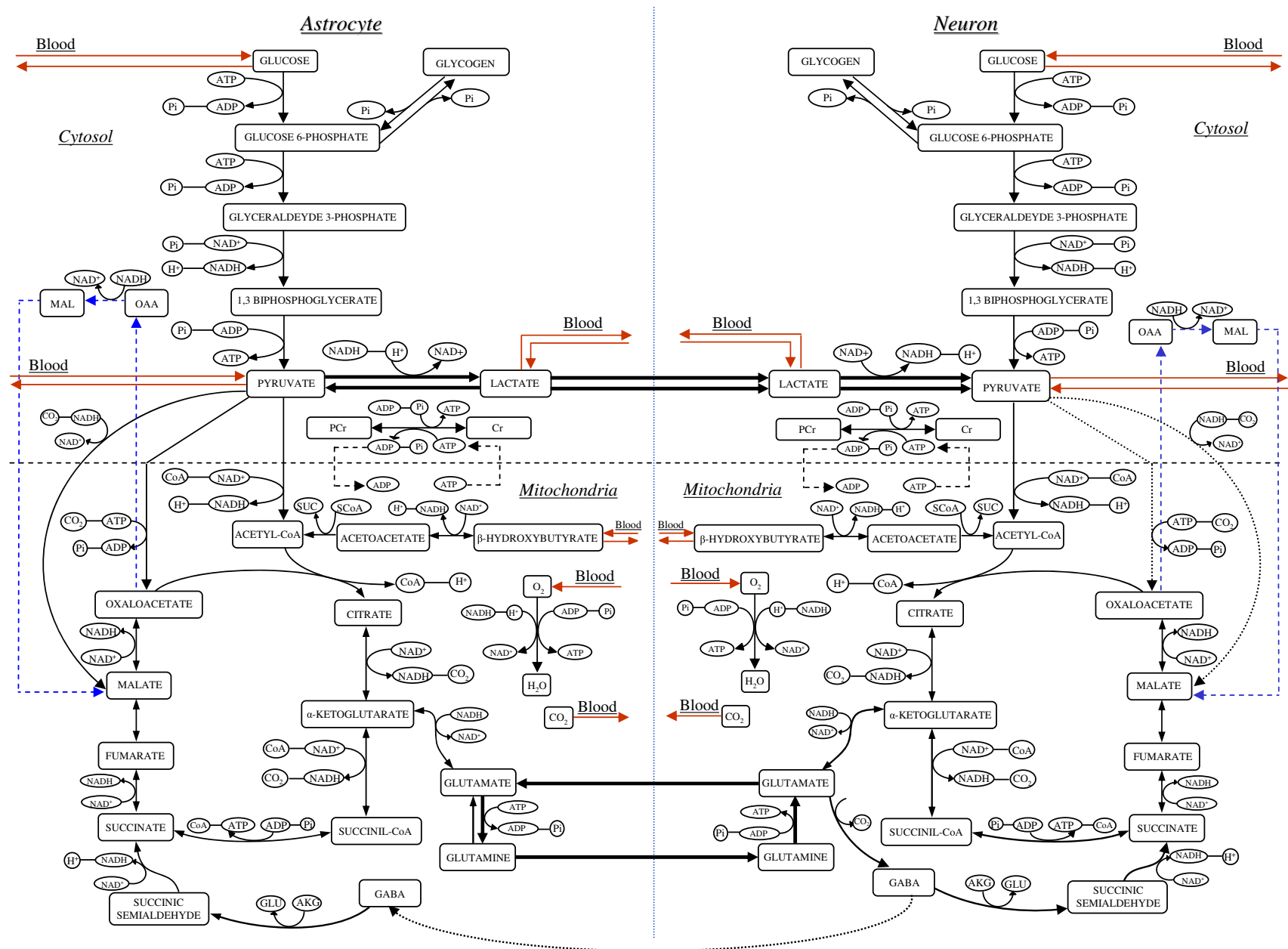

FIGURE 1. A schematic picture of the biochemical pathways included in the present model. Details concerning the stoichiometry can be found in Appendix B.

tistical approach to the FBA, providing a versatile framework where various constraints motivated by a priori biochemical or physiological information, as well as by experimental data, can be incorporated into the model. We will also show that starting from a general, detailed and thus complex model, the statistical approach to FBA can automatically find those unknowns that are statistically correlated thus removing the need for additional lumping of the model.

\section{A FIVE-COMPARTMENT BRAIN METABOLIC MODEL}

To model and simulate the metabolic processes in the brain under different energetic states and to take into consideration the contributions of the different compartments, it is important to develop a general mathematical model which includes many of the important reactions and exchanges. A comprehensive model of brain energy metabolism which takes into account most of the major biochemical processes has not yet been described in the literature. Several models distinguish between cell types (astrocyte and neuron), but only include some aspects of brain energy metabolism. For example, the model of Aubert et al. focuses on the Astrocyte-Neuron Lactate Shuttle, and the one by Patel et al. on the glutamate-glutamine cycle, lumping most of the intermediate biochemical mechanisms of the TCA cycle and of the glycolysis. ${ }^{1,18,19}$ While this is justified in studies focusing on some specific aspects of brain metabolism for which measured data is available, it nonetheless does not diminish the value of an integrated model capable of capturing the complexity of the system.

In this work we propose a five-compartment model of brain metabolism, which incorporates two types of brain cells, astrocytes, and neurons, and for each one of them a cytosolic and mitochondrial compartmentalization with intermediate metabolites. This model follows and generalizes the paradigm of the myocardial 
metabolism model proposed by Zhou et al. ${ }^{28}$ The five compartments considered in our model are the cytosol and mitochondria for astrocyte and neuron, and the blood domain, which includes interstitial fluid. Since this model will provide the basis for several in silico experiments, it includes detailed descriptions of glycolysis, TCA cycles, glutamine-glutamate and GABA shunts for both cell types as well as substrate transports between the domains. The diagram of this fivecompartment model is shown in Fig. 1.

In the following subsection we give a general description of the five-compartment brain model, which will be used in subsequent work to study the effects of perturbed metabolic pathways as a result of utilization of different energy substrates.

\section{The General Model}

Each one of the two cerebral cell types considered by our model, astrocyte and neuron, are subcompartmentalized into cytosol and mitochondria. In each cytosolic domain there are 16 biochemical species, while we distinguish 23 species in each mitochondrial domain and 6 in the blood domain. Of these biochemical species, glucose, pyruvate, and lactate are interchanged between blood and cytosol. The exchange fluxes of $\beta$-hydroxybutyrate, $\mathrm{O}_{2}$ and $\mathrm{CO}_{2}$ between blood and cytosol and further between cytosol and mitochondria are lumped together to fluxes between blood and mitochondria because of lack of cytosolic reactions involving these compounds. The same type of lumping is done for the neurotransmitter fluxes. We model also the exchange fluxes of lactate, glutamate, glutamine, and GABA between astrocyte and neuron.

In addition to the standard glycolytic flux, TCA cycle, phosphocreatine synthesis and oxidative phosphorylation, our model includes the ANLS, that plays a central role in the hypothesis that lactate is transported from astrocyte to neuron according to the neuronal demand. ${ }^{17}$ As indicated in the schematics of the biochemical pathways in Fig. 1, in the present model both TCA cycles (astrocyte and neuron) interact with the neurotransmitters through the glutamateglutamine cycle and the GABA shunt. ${ }^{9,25}$ Furthermore, the model allows anaplerotic reactions between TCA cycle and pyruvate in astrocyte and in neuron. ${ }^{8}$

To study the dynamics of brain metabolism, the change in the concentrations of the biochemical species in each compartment over time is modeled by a system of ordinary differential equations based on mass balance equations. Each equation expresses the change in concentration of a species through three different mechanisms: convection, transport and reaction, which do not all need to be present in the same equation. In fact, since in the blood domain no chemical reactions occur, the reaction term vanishes for the equations in the blood, which depend only on convection and transport between the blood and the cell domains. The dynamic mass balance equation for a species $j$ in the blood domain can be written as

$$
\begin{aligned}
V_{b} \frac{d \mathbf{C}_{b, j}}{d t}= & \underbrace{\frac{Q\left(C_{a, j}-C_{b, j}\right)}{F}}_{\text {Convection }} \\
& +\underbrace{J_{c A \rightarrow b, j}-J_{b \rightarrow c A, j}+J_{c N \rightarrow b, j}-J_{b \rightarrow c N, j}}_{\text {Transport }} \\
& +\underbrace{J_{m A \rightarrow b, j}-J_{b \rightarrow m A, j}+J_{m N \rightarrow b, j}-J_{b \rightarrow m N, j}}_{\text {Transport }},
\end{aligned}
$$

where $V_{b}$ is the domain volume, $F$ is the mixing fraction, $Q$ is the blood flow, $C_{a, j}$ is the arterial concentration of species $j$, and $C_{v, j}$ is the venous concentration of species $j$. The terms $J_{c A} \rightarrow b, j$ and $J_{b} \rightarrow c A, j$ are the transport rates between blood and cytosol in astrocyte, and $J_{c N} \rightarrow b, j$ and $J_{b \rightarrow c N, j}$ are the transport rates between blood and neuronal cytosol. Similarly, the terms $J_{m A} \rightarrow b, j, J_{b} \rightarrow m A, j$ and $J_{m N} \rightarrow b, j, J_{b} \rightarrow m N_{, j}$ are the transport rates between blood and the two mitochondria, respectively. Notice that in reality the transport between blood and mitochondria occurs via cytosol as explained above.

In the cytosolic and mitochondrial domains, the concentration of a species may change as a result of metabolic reactions and mass transport across the cellular and mitochondrial membrane. Thus, the dynamic mass balance equation for a species $j$ in the cytosol or mitochondria domain in astrocyte is of the form

$$
\begin{aligned}
V_{z A} \frac{d C_{z A, j}}{d t}= & \underbrace{R_{z A, j}}_{\text {Reaction }} \\
& +\underbrace{J_{b \rightarrow z A, j}-J_{z A \rightarrow b, j}+J_{m A \rightarrow c A, j}-J_{c A \rightarrow m A, j}}_{\text {Transport }} \\
& +\underbrace{J_{z N \rightarrow z A, j}-J_{z A \rightarrow z N, j}}_{\text {Transport }}, \quad z=c \text { or } m,
\end{aligned}
$$

where $R_{z A, j}$ is the metabolic reaction rate, $V_{z A}$ is the relative volume of the cytosol or mitochondrial domain in astrocyte, $J_{m A \rightarrow c A, j}$ and $J_{c A \rightarrow m A, j}$ are the transport rates between mitochondria and cytosol in astrocyte. The terms $J_{z N} \rightarrow z A, j$ and $J_{z A} \rightarrow z_{N N, j}$ are the transport rates between cytosol/mitochondria in neuron and in astrocyte, respectively.

Similarly, the dynamic mass balance equation for a species $j$ in the cytosol or mitochondria domain in neuron is of the form 


$$
\begin{aligned}
V_{z N} \frac{d C_{z N, j}}{d t}= & \underbrace{R_{z N, j}}_{\text {Reaction }} \\
& +\underbrace{J_{b \rightarrow z N, j}-J_{z N \rightarrow b, j}+J_{m N \rightarrow c N, j}-J_{c N \rightarrow m N_{, j}}}_{\text {Transport }} \\
& +\underbrace{J_{z A \rightarrow z N, j}-J_{z N \rightarrow z A, j}}_{\text {Transport }}+\underbrace{T_{z N, j}}_{\text {Activity }}, \quad z=c \text { or } m,
\end{aligned}
$$

with obvious notation of the transport fluxes. The term $T_{z N, j}$ of neural activity is an essential novelty of the present model. It accounts for the energy demand of the neuron, due to sustain the glutamate-glutamine and GABA exchange between the astrocyte and neuron. In the present model, we assume that $T_{z N, j}$ vanishes for all mass balance equations except for the ATP, ADP and phosphate in mitochondria of the neuron. The present implementation is based on the assumption that mitochondrial ATP of the neuron is consumed to maintain the glutamate and GABA fluxes into the intercellular synaptic gap, leading to a model

$$
\begin{aligned}
T_{m N, \mathrm{ATP}} & =-\alpha J_{N \rightarrow A, \mathrm{GLU}}-\beta J_{N \rightarrow A, \mathrm{GABA}} \\
& =-T_{m N, \mathrm{ADP}}=-T_{m N, P i}
\end{aligned}
$$

The coefficients $\alpha$ and $\beta$ are estimated based on the experimental information that to transport one unit of glutamate, $68 \%$ of the cellular metabolic rate is required. ${ }^{9}$ This rate is identified with the total ATP turnover by glycolysis and oxidative phosphorylation in the neuron. ${ }^{7}$ Glycolysis produces two units of ATP and oxidative phosphorylation 36 , so we estimate the coefficient $\alpha$ to be $\alpha=0.68 \times 38=25.84$. Similarly, the GABA flux requires approximately $21 \%$ of ATP flux, yielding an estimate $\beta=0.21 \times 38=7.98$.

The net metabolic reaction rate of a species $j$ in compartment $\ell, R_{\ell, j}$, can be expressed as the difference between the rate of species production $P_{\ell, j}$ and the rate of species utilization $U_{\ell, j}$,

$$
R_{\ell, j}=P_{\ell, j}-U_{\ell, j}=\sum_{k} \beta_{j, k}^{\ell} \Phi_{k}^{\ell}-\sum_{k} \widetilde{\beta}_{j, k}^{\ell} \Phi_{k}^{\ell} .
$$

The summation index $k$ runs over all reactions taking place in the $\ell$ th compartment, $\Phi_{k}^{\ell}$ is the reaction flux corresponding to the $k$ th reaction in compartment $\ell$, and the terms $\beta_{j, k}^{\ell}$ and $\widetilde{\beta}_{j, k}^{\ell}$ are the corresponding stoichiometric coefficients.

Under steady state conditions, the concentrations do not change, hence the time derivatives must vanish. Setting the left hand side of Eqs. (1)-(3) to zero, we obtain a system of linear equations involving the reaction and transport fluxes, and the arterial and venous blood concentrations. While the arterial concentrations may be assumed to be known, the venous ones cannot, since they depend on the cellular intake of metabolites. Hence, the venous concentrations are treated as unknowns in our system. We remark that since the model depends only on the difference between arterial and venous concentrations, the arterial value can be thought of as a baseline. By measuring both arterial and venous values, we have another estimate for the difference. This is equivalent to assume that we have measured venous value around a fixed baseline with variance equal to the sum of the variances of the venous and arterial values.

We collect all 75 reaction fluxes into a column vector $\Phi$, the 34 transport fluxes are collected in the column vector $\mathbf{J}$ and the six venous concentrations in the vector $\mathbf{C}_{b}$. These vectors of unknowns are then stacked into a column vector $\mathbf{u}=\left[\Phi, \mathbf{J}, \mathbf{C}_{b}\right]$ of length 115 , which is the primary unknown in our model. The steady state equations obtained by setting to zero the derivatives in (1)-(3) are expressed in matrix form as

$$
A \mathbf{u}=\mathbf{r},
$$

where $A$ is a $84 \times 115$ matrix and $\mathbf{r}$ is a column vector, comprising of zeros corresponding to Eqs. (2) and (3), and the scaled arterial concentrations $-Q C_{a, j} / F$ corresponding to the Eq. (1) in the blood domain.

In addition to the linear system (4), the fluxes need to satisfy a set of inequality constraints which ensure, among other things, that they are meaningful. The constraints include non-negativity, possible upper bounds as saturation values of some reactions and fluxes and lower bounds to control the activity level in the cell. ${ }^{12,13}$ Note that the limited supply of glucose and oxygen set natural upper bounds for the reactions, thus relieving the need for imposing additional constraints. The bound constraints will be specified later when the computed results are discussed. At this point, we simply write them as a vectorial inequality

$$
C \mathbf{u} \geq \mathbf{c}
$$

where the inequality is understood componentwise, and the matrix $C$ and the vector $\mathbf{c}$ are constructed so that each row in (5) corresponds to one inequality constraint.

\section{The Baseline Model}

In baseline condition, we do not expect all possible reaction and transfer pathways to be active. Therefore, the study of the brain metabolic functions under these conditions can be carried out by a slightly simplified model where some of the non-active pathways are blocked. More specifically, the following reduction has been applied to the general model (Fig. 1): 
- Ketones are essentially absent in baseline standard (steady state) conditions. Therefore the transfer flux from blood to tissue of the $\beta$-hydroxybutyrate is set to zero. This in turn implies that acetoacetate fluxes are also absent.

- TCA cycles in baseline conditions are assumed to function in a preferred direction, thus their bidirectional reaction fluxes are replaced by positive net reaction fluxes in the preferred direction.

- The anaplerotic reactions pyruvate $\longrightarrow$ malate and pyruvate $\longrightarrow$ oxaloacetate are absent in neuron in the baseline model but possible in the astrocyte.

- Pyruvate reduction in neuron is neglected since we follow the ANLS hypothesis.

- Glycogen synthesis and breakdown at steady state, both in astrocyte and neuron, are assumed to be in equilibrium. Therefore, since the net flux is zero it is ignored.

In the current FBA we used the baseline model, implementing the described reduction from the general model by setting the reaction fluxes and transport rates which are expected to be inactive to zero. Our model is therefore flexible enough to easily allow the inclusion of presently blocked pathways.

\section{METHODS}

We model a metabolic system as a complex network of biochemical pathways. Traditionally, the FBA of a given network consists of finding feasible flux values that satisfy the flux balance equations under steady state conditions. The linear system of the steady state equations for the fluxes is usually underdetermined, and its solution must be non-negative. Therefore the solution of the FBA must satisfy

$$
A \mathbf{u}=\mathbf{b}, \quad C \mathbf{u} \geq \mathbf{c},
$$

where $\mathbf{u} \in \mathbb{R}^{n}$ is the vector of unknowns containing the reaction fluxes and transport rates, $A \in \mathbb{R}^{m \times n}$ is the system matrix, which typically has fewer rows than columns, $\mathbf{b} \in \mathbb{R}^{m}$ is the input vector, and the inequality constraints for the unknown $\mathbf{u}$ are organized in the arrays $C \in \mathbb{R}^{k \times n}, \mathbf{c} \in \mathbb{R}^{k}$

Solving the linear system (6) in the classical sense may be unfeasible for several reasons. In fact, since the matrix $A$ has fewer rows than columns, the system is underdetermined and may therefore admit infinitely many feasible solutions. While requiring that the unknowns satisfy some constraints may restrict the solutions space, imposing too many inequality constrains easily leads to a system having no feasible solution. For these reasons, traditional approaches based, e.g., on optimization, may find solutions that depend entirely on the solution method rather than the properties of the system, or may fail to find a solution at all. To avoid these shortcomings all together, we approach the FBA problem from the point of view of statistical inference.

\section{Flux Balance Analysis as Quest for Information}

The point of view of Bayesian statistics is to model the unknowns as random variables, the randomness being a model for our lack of information about their values. The model ties the unknowns to observed variables which may themselves contain uncertainties. The classical estimation problem is then replaced by a statistical inference problem: how much information about the unknown vector the observations and the prior information give? The information is encoded into probability distributions. ${ }^{10}$

Following the Bayesian paradigm, we interpret the vector $\mathbf{u}$ containing the reaction and transfer fluxes as a random variable whose a priori probability density is $\pi_{\text {prior }}(\mathbf{u})$. Since we assume that the bound constraints are known, we choose a prior density of the form

$$
\pi_{\text {prior }}(\mathbf{u}) \propto \Theta(C \mathbf{u}-\mathbf{c}),
$$

where ' $\alpha$ ' means 'equal up to a multiplicative norming constant' and $\Theta$ is a multivariate Heaviside function, which takes on the value one if all components of the argument are non-negative and vanishes otherwise.

To encode the linear equality constraint in the model, we interpret the right side $\mathbf{b}$ as an observation that may contain noise. An additive noise model is used here, i.e.,

$$
\mathbf{b}=A \mathbf{u}+\mathbf{e}
$$

where $\mathbf{e} \in \mathbb{R}^{m}$ is a random noise vector. Observe that in the present model, the vector $\mathbf{b}$ consists of zeroes corresponding to the steady state flux balance equations within the cells and of estimated arterial concentrations. The assumption that the arterial concentrations are contaminated by additive noise, means that these values are treated as observations that may vary over a population. The noise vector added to the zeroes corresponding to the internal flux balance conditions expresses a lack of certainty whether the system is really at steady state. The noise may account also for uncertainties in the pathway network model. Notice that we refer to the zeroes in the flux balance equations as observations, while in reality they are implicitly assumed to give a zero output, if we had a way to measure them. In fact we may think of them as virtual observations. 
Assuming that the probability distribution $\pi_{\text {noise }}$ of the noise vector $\mathbf{e}$ is known, we may write the likelihood density as

$$
\pi(\mathbf{b} \mid \mathbf{u}) \propto \pi_{\text {noise }}(\mathbf{b}-A \mathbf{u})
$$

This density expresses the probability distribution of the noisy observation vector under the condition that the value of $\mathbf{u}$ is known and fixed.

In our FBA, we are interested in the opposite question: What is the probability distribution of the unknown $\mathbf{u}$ assuming that $\mathbf{b}$ is known and fixed at the observed value? The answer is the posterior probability density that, according to Bayes' theorem, can be expressed as

$$
\pi(\mathbf{u} \mid \mathbf{b}) \propto \pi_{\text {prior }}(\mathbf{u}) \pi(\mathbf{b} \mid \mathbf{u}), \quad \mathbf{b}=\mathbf{b}_{\text {observed }}
$$

The posterior probability density is the solution of the statistical FBA problem: it expresses, in terms of probability distributions, how much information the prior, the model and the observation give us about the unknown quantities.

As usual in statistical inference problems, the major computational challenge is the numerical exploration of the posterior probability densities. The method of choice is to use sampling techniques, in particular MCMC methods, which produce a large ensemble of specimen vectors $\mathbf{u}_{j}, 1 \leq j \leq N$, distributed according to the posterior probability density $\pi(\mathbf{u} \mid \mathbf{b}) .{ }^{5,9,14}$ Single estimates can be computed based on the sample, the most important from our point of view being the posterior mean estimate,

$$
\mathbf{u}_{\mathrm{pm}}=\int_{\mathbb{R}^{n}} \mathbf{u} \pi(\mathbf{u} \mid \mathbf{b}) d \mathbf{u} \approx \frac{1}{N} \sum_{j=1}^{N} \mathbf{u}_{j}=\overline{\mathbf{u}}_{\mathrm{pm}}
$$

or, equivalently, the expectation of $\mathbf{u}$ with respect to the posterior probability density. Similarly, we estimate the estimation error covariance matrix,

$$
\begin{aligned}
\operatorname{cov}(\mathbf{u}) & =\int_{\mathbb{R}^{n}}\left(\mathbf{u}-\mathbf{u}_{\mathrm{pm}}\right)\left(\mathbf{u}-\mathbf{u}_{\mathrm{pm}}\right)^{\mathrm{T}} \pi(\mathbf{u} \mid \mathbf{b}) d \mathbf{u} \\
& \approx \frac{1}{N} \sum_{j=1}^{N}\left(\mathbf{u}_{j}-\overline{\mathbf{u}}_{\mathrm{pm}}\right)\left(\mathbf{u}_{j}-\overline{\mathbf{u}}_{\mathrm{pm}}\right)
\end{aligned}
$$

The diagonal of this matrix gives estimates for the variances and the standard deviations for the components. Projections of the posterior density can be represented as histograms of the components of the sample. A schematic representation of the sampling and the related one-dimensional marginal histograms is shown in Fig. 2.

In Appendix A, we explain the MCMC algorithm employed in this work to produce the sample.

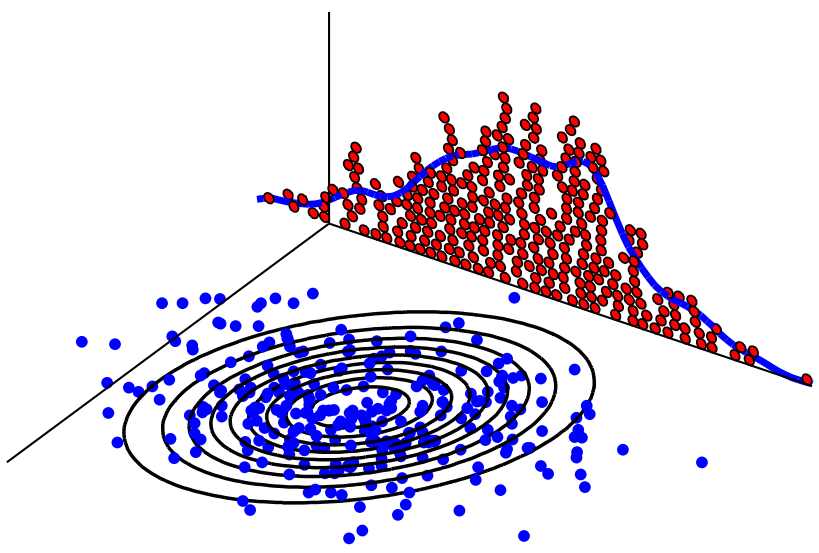

FIGURE 2. A schematic representation of sampling and the interpretation of the histograms of marginal distributions. The two-dimensional distribution is represented as a contour plot. The marginal distribution is estimated as a histogram of the second components of the sample.

\section{RESULTS}

In this section, we describe which questions can be answered with our FBA for the five-compartment model for brain metabolism proposed in Sect. "A FiveCompartment Brain Metabolic Model." The conclusions of our investigation are then compared with experimental data and with the hypotheses presented in literature. , $8,9,17,19,20,24$ Modifications of the model are proposed to make the results conform with what is known about brain metabolism.

\section{Statistical Analysis of Metabolic Pathways}

The two main questions leading our computational experiments are:

- Which metabolic pathways are more active in brain metabolism at steady states with different levels of neural activity?

- Which constraints have to be applied in order for our model to be in agreement with what can be inferred about brain metabolism based on experimental data?

To answer these questions, we made a preliminary tuning run of 200,000 sample points of the MCMC algorithm using the general metabolic model described above. This step required a couple of hours on a standard PC (Pentium 4, 2 Gb memory) using MATLAB. Lower bounds for the glutamate and GABA fluxes from neuron to astrocyte were used as control parameters to simulate the intensity of neural activity. In our computed experiments, we consider two activity intensities that we refer to as low and high neural activity. The bounds characterizing the low neural activity are 


$$
\begin{aligned}
& J_{m N \rightarrow m A, \mathrm{GLU}} \geq \ell_{\mathrm{low}, \mathrm{GLU}}=0.2, \\
& J_{m N \rightarrow m A, \mathrm{GABA}} \geq \ell_{\mathrm{low}, \mathrm{GABA}}=0.066
\end{aligned}
$$

while those characterizing high neural activity are ${ }^{24}$

$$
\begin{aligned}
& J_{m N \rightarrow m A, \mathrm{GLU}} \geq \ell_{\text {high, } \mathrm{GLU}}=0.8, \\
& J_{m N \rightarrow m A, \mathrm{GABA}} \geq \ell_{\text {high, GABA }}=0.24 .
\end{aligned}
$$

The standard deviation $\sigma$ in the covariance matrix of the likelihood is set to $\sigma=1 \times 10^{-3}$, and the standard deviation of the observed arterial values is $n=5 \%$ (see Appendix A, formula (14)). In our model, we used the value $Q / F=1.5 \mathrm{~L} / \mathrm{min}$. The arterial blood values, in units $\mathrm{mmol} / \mathrm{L}$, were $C_{\mathrm{a}, \mathrm{GLC}}=6.0, C_{\mathrm{a}, \mathrm{O}_{2}}=5.18$, $C_{\mathrm{a}, \mathrm{CO}_{2}}=19.5, C_{\mathrm{a}, \mathrm{PYR}}=0.66$ and $C_{\mathrm{a}, \mathrm{LAC}}=7.0 .{ }^{15,16}$

The analysis of the results of the tuning run indicated that the full model showed some disagreement with known results about brain metabolism. In particular:

- The fluxes in the GABA shunt in neuron was much stronger than the TCA cycle, thus contradicting the common understanding of the functioning of the neuron. To correct this unbalance, we blocked the flux SUC $\longrightarrow$ SCoA in the TCA cycle, thus effectively implying that the net flux is in the opposite direction or, equivalently, that the normal TCA direction becomes dominant.

- If no constraints are applied in the general model, the neuron prefers to take large amount of pyruvate from the blood domain, rather than from neuronal glycolysis or through the ANLS. Although FBA does not give direct information of the substrate concentrations, there are good reasons to believe that such a situation leads to an anomalous redox shift, pp. $419^{25,27}$. To avoid this situation, we simulate the pyruvate-lactate balance by imposing the constraint

$$
J_{b \rightarrow c N, \mathrm{PYR}} \leq 0.1 J_{A \rightarrow N, \mathrm{LAC}} .
$$

- The flow of lactate from neuron to blood was found to be high. To avoid lactate losses, we closed the lactate channels between blood and both cell types.

After these adjustments to the model, we rerun the MCMC algorithm with 200,000 sample points at both low and high neural activity. In the following, we summarize the results.

The key quantities estimated from the samples are

1. Glycolytic fluxes in astrocyte and neuron;

2. Lactate shuttle from astrocyte to neuron;

3. TCA cycles in astrocyte and neuron;
4. Oxidative phosphorylation in astrocyte and neuron;

5. GABA shunt in neuron;

6. Glutamine-glutamate shuttle between the cell types.

We characterize these quantities using the posterior mean estimates. We test the convergence rate of the posterior mean estimate by checking how strongly correlated the components in the samples are. In Figs. 3 and 4 we show some of the sample histories, with a burn-in sequence of length 20,000 left out from the start of the chain.

Without going into a detailed diagnosis of the convergence of the posterior mean, the sample histories in Fig. 3 indicate a good convergence and therefore reliable estimate of the posterior means in the astrocyte. The sample histories in Fig. 4 show a larger correlation and therefore less reliable estimation results of the posterior mean. Notice that there is a strong correlation between the TCA flux (ACoA $\longrightarrow$ CIT), the GLU $\longrightarrow$ GABA flux and the oxidative phosphorylation flux in the neuron (Table 1).

We compare the results corresponding to high and low neural activity by plotting some of the sample histograms for both low and high activity levels in Fig. 5. The histograms of oxidative phosphorylation and glycolysis seem to follow roughly a scaling law: as the neural activity increases fourfold, the histogram shifts with a factor close to four.

The results of our FBA are summarized in the diagrams shown in Figure 6, where the quantities characterizing the neural activities are also indicated. It is clear from our in silico experiments that the oxidative phosphorylation ratio,

$$
r_{\mathrm{OxPhos}}=\frac{\Phi_{\mathrm{O}_{2} \rightarrow \mathrm{H}_{2} \mathrm{O}, N}}{\Phi_{\mathrm{O}_{2} \rightarrow \mathrm{H}_{2} \mathrm{O}, A}}
$$

is of the order 100 with both activation levels. The GABA shunt in both cases is about two thirds of the neuronal TCA cycle, and the V-cycle is about one half of the TCA cycle. The TCA cycle in neuron is orders of magnitudes higher than in astrocyte.

A result which is in contradiction with what reported in the literature is that glycolysis is of about the same order of magnitude in both cell types, and at high neural activity level, is higher in neuron than in astrocyte. While this might be the case at low activity levels, as verified in some in vitro experiments, it is commonly accepted that the main source of carbons for the neuron is the lactate flux from the astrocyte. ${ }^{4,7,17}$

An explanation for this discrepancy may be that our model does not take into account that astrocytes and neurons may have different exposure to glucose, 

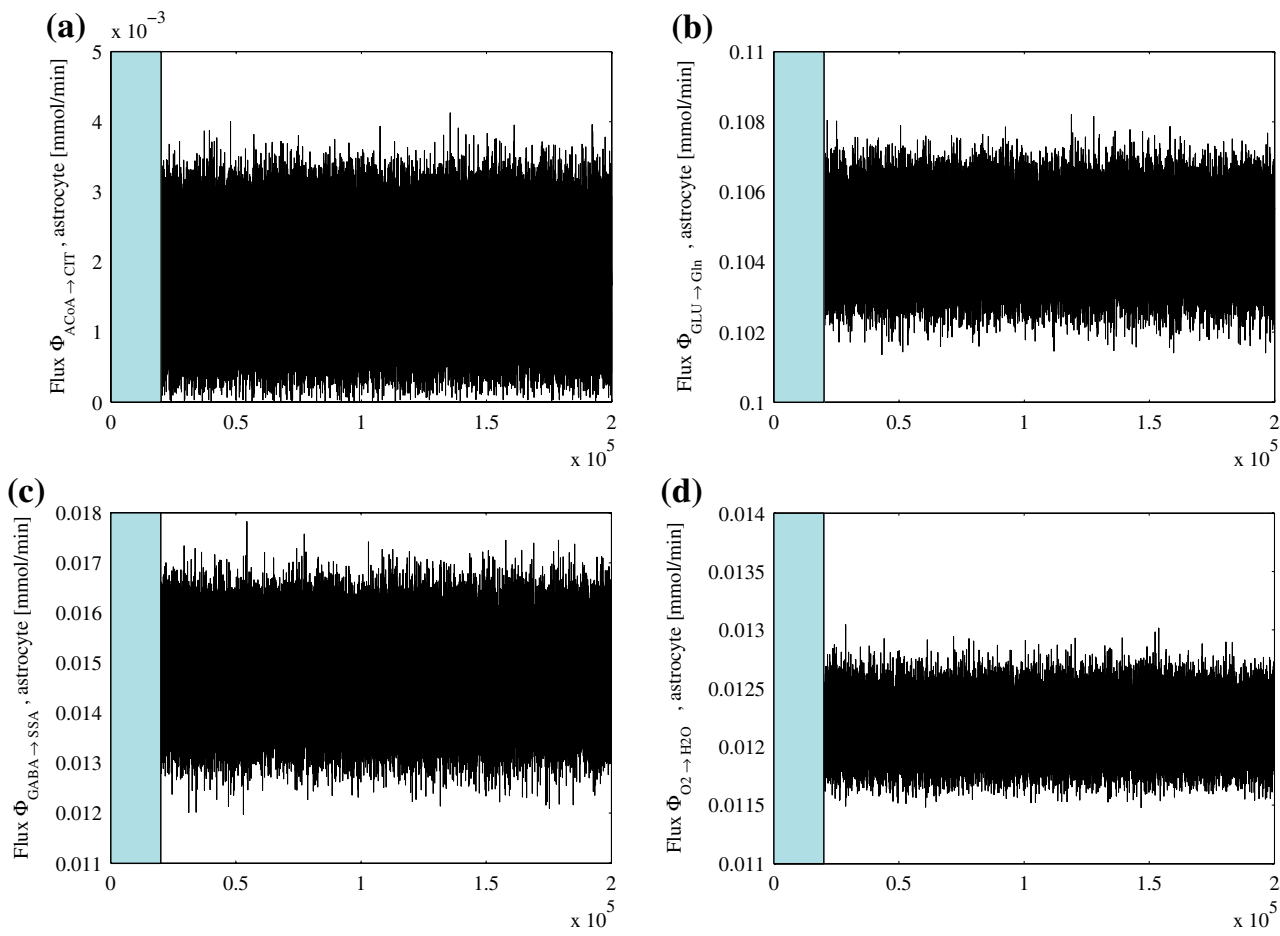

FIGURE 3. Sample histories of selected fluxes in the astrocyte corresponding to the low neural activity model. The shaded area indicates the removed burn-in sequence of 20,000 points.
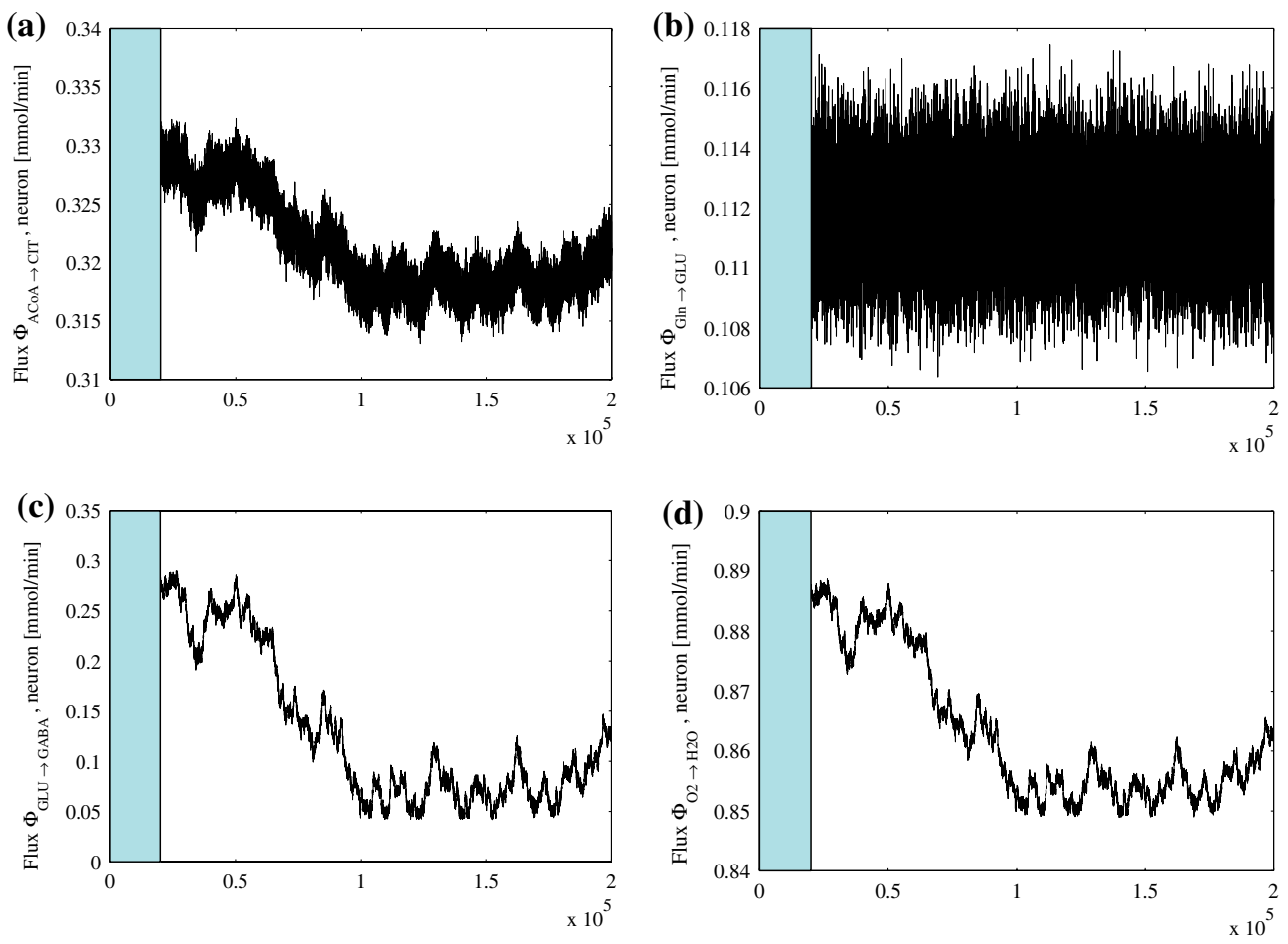

FIGURE 4. Sample histories of selected fluxes in the neuron corresponding to the high neural activity model. 
TABLE 1. Posterior mean values \pm standard deviations (STD) predicted by our model for the most relevant biochemical reactions and transport fluxes for different neural activation levels when we do not regulate the glucose influx.

\begin{tabular}{|c|c|c|c|c|}
\hline \multirow[b]{3}{*}{ Activity } & \multicolumn{4}{|c|}{ Unregulated glucose influx } \\
\hline & \multicolumn{2}{|c|}{0.2} & \multicolumn{2}{|c|}{0.8} \\
\hline & A & $\mathrm{N}$ & A & $\mathrm{N}$ \\
\hline$J_{b \rightarrow c, \mathrm{GLC}}$ & $(1.12 \pm 0.01) \times 10^{-1}$ & $(8.07 \pm 2.30) \times 10^{-2}$ & $(3.22 \pm 3.40) \times 10^{-1}$ & $(4.63 \pm 0.17) \times 10^{-1}$ \\
\hline$\Phi_{\mathrm{PYR} \rightarrow \mathrm{G} \rightarrow \mathrm{ACOA}}$ & $(4.53 \pm 4.61) \times 10^{-5}$ & $(3.26 \pm 0.04) \times 10^{-1}$ & $(1.11 \pm 1.13) \times 10^{-5}$ & $1.33 \pm 0$ \\
\hline$\Phi_{\mathrm{CIT} \rightarrow \mathrm{AKG}}$ & $(2.10 \pm 2.14) \times 10^{-5}$ & $(3.36 \pm 0.04) \times 10^{-1}$ & $(5.19 \pm 5.27) \times 10^{-6}$ & $1.37 \pm 0$ \\
\hline$\Phi_{\mathrm{SUC} \rightarrow \mathrm{MAL}}$ & $(4.21 \pm 4.28) \times 10^{-5}$ & $(2.83 \pm 0.04) \times 10^{-1}$ & $(1.05 \pm 1.07) \times 10^{-5}$ & $1.16 \pm 0$ \\
\hline$\Phi_{\mathrm{GLU} \rightarrow \mathrm{GABA}}$ & & $(1.29 \pm 0.75) \times 10^{-1}$ & & $1.07 \pm 0.03$ \\
\hline$\Phi_{\mathrm{O}_{2} \rightarrow \mathrm{H}_{2} \mathrm{O}}$ & $(1.22 \pm 0.01) \times 10^{-2}$ & $(8.63 \pm 0.12) \times 10^{-1}$ & $(4.84 \pm 0.01) \times 10^{-2}$ & $3.54 \pm 0$ \\
\hline$J_{A \rightarrow N, \text { LAC }}$ & \multicolumn{2}{|c|}{$(2.24 \pm 0.49) \times 10^{-1}$} & \multicolumn{2}{|c|}{$(6.45 \pm 0.34) \times 10^{-1}$} \\
\hline$J_{N \rightarrow A, G L U}$ & \multirow{2}{*}{\multicolumn{2}{|c|}{$\frac{0.2}{(108+0.1) \times 10^{-1}}$}} & \multicolumn{2}{|c|}{0.8} \\
\hline$J_{A \rightarrow N, G l n}$ & & & \multirow{2}{*}{\multicolumn{2}{|c|}{$\begin{array}{c}(4.32 \pm 0.01) \times 10^{-1} \\
0.24\end{array}$}} \\
\hline$J_{N \rightarrow A, G A B A}$ & \multicolumn{2}{|c|}{0.06} & & \\
\hline
\end{tabular}

STD $=0$ means that the computed STD is less than the round off error. The value for $J_{b \rightarrow c, \text { GLC }}$ is a net transport and the value for $\Phi_{\text {SUC } \rightarrow \text { MAL }}$ is the average of the two metabolic fluxes $\Phi_{\mathrm{SUC} \rightarrow \mathrm{FUM}}$ and $\Phi_{\mathrm{FUM} \rightarrow \mathrm{MAL}}$.

implying an uneven breakdown of the glucose influx. To signal that astrocyte and neuron may have different access to glucose, we introduce an additional bound constraint of the form

$$
J_{b \rightarrow c N, \mathrm{GLC}} \leq \tau J_{b \rightarrow c A, \mathrm{GLC}}, \quad \tau<1,
$$

and we set here a rather loose bound, $\tau=0.5$. Additional bounds tend to slow down the convergence of the Gibbs sampler, so to facilitate the convergence of the MCMC, we increase the standard deviation in the likelihood function to $\sigma=0.1$. Based on these samples, we estimate again the posterior means of the fluxes. Some of the values characterizing the activities are summarized in the pathway diagram shown in Fig. 7. The changes compared to the values with no glucose flux bounds are in better agreement with the results in the literature (Table 2).

\section{CONCLUSIONS AND FUTURE WORK}

This work is concerned with a recently introduced versatile statistical approach to the classical FBA of metabolic networks, and it is applied to a new five-compartment brain model which includes two cell types with intracellular compartmentalization and a control on the neural activity level. We have shown that although the general model is quite complicated, it is possible to interpret the results in terms of simplified network, as shown in Figs. 6 and 7, without additional lumping. The key results predicted by the FBA for our model, illustrated in those figures and Tables 1 and 2, can be summarized as follows.

- Oxidative phosphorylation in neuron is essentially insensitive to neuronal glucose influx, but it is proportional to the neural activity;

- The fractional proportion of glucose influx does not affect the TCA cycle in neuron, but it has a marked effect on the TCA cycle in astrocyte;

- Glycolysis is equally active in both cell types when equal fractional proportions of glucose influx are available to astrocyte and neuron.

- The TCA cycle is orders of magnitude slower in astrocyte than in neuron when equal fractional proportions of glucose influx are available to both cell types.

- The V-cycle and the GABA shunt are not overly sensitive to changes in the fractional proportions of glucose influx to astrocyte and neuron.

- The flux values in the GABA shunt are greater than those of the V-cycle. Whether this is physiologically relevant is unknown and will require further investigation.

In this work we have shown that our approach makes it possible to identify pathways that the system prefers for its fuel supply based on FBA, and to pinpoint reaction fluxes that need to be regulated for the predicted results to be in accordance with biochemically and physiologically motivated hypotheses as well as with experimentally verified phenomena. Hence, the new paradigm for modelling metabolic systems and the statistical approach to FBA are useful 

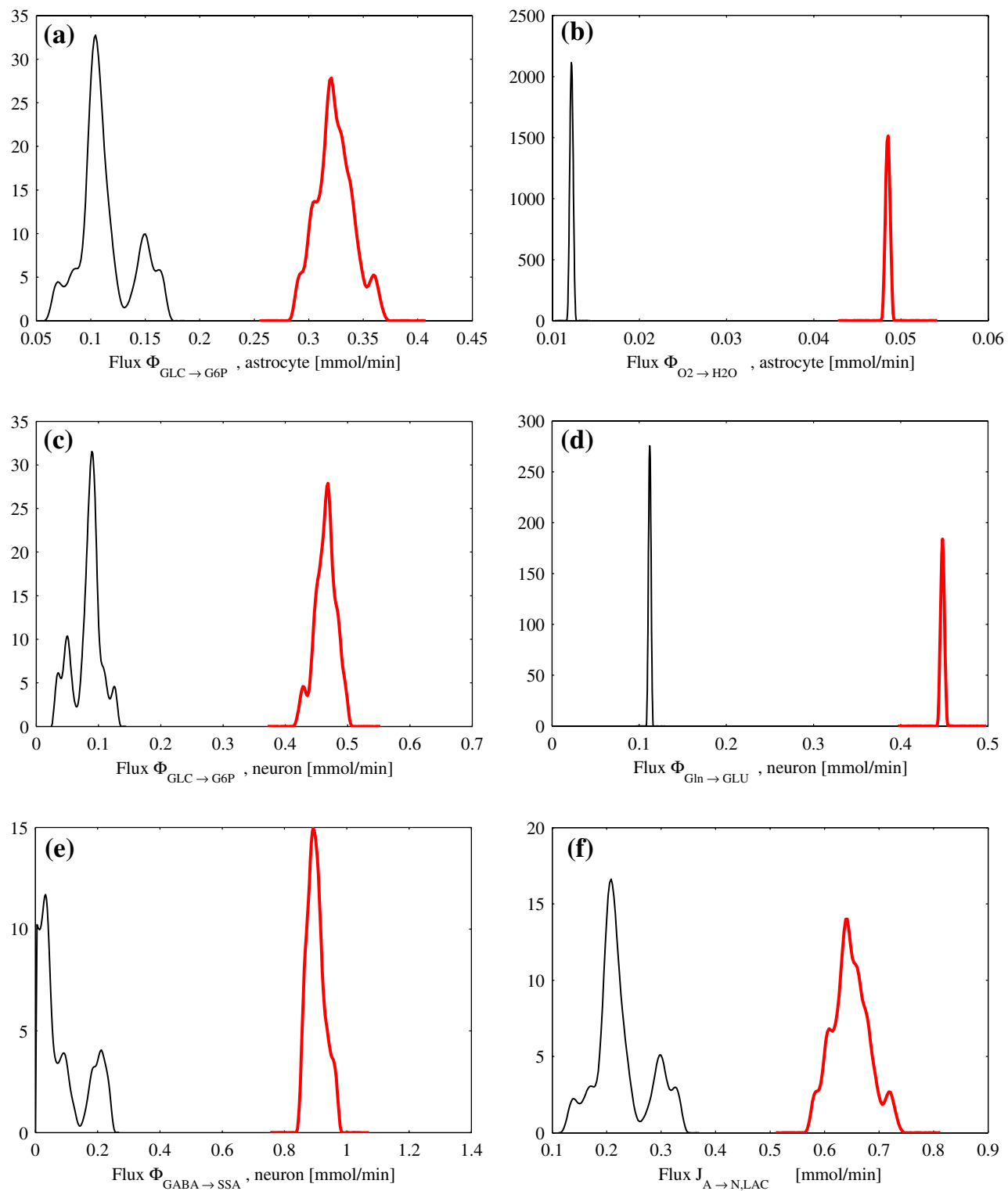

FIGURE 5. Sample histograms corresponding to the low activity model (thin black) and high activity model (thick red).

in redesigning the metabolic networks as well as to test hypotheses concerning the activation of different pathways. Future studies will consider more complex models which separate GABAergic and glutamatergic neurons as well as include more sophisticated descriptions of the shuttle mechanisms between astrocytes and neurons and within each individual cell type.

\section{APPENDIX A: MCMC BY GIBBS SAMPLER}

In this Appendix, we describe the implementation of the Markov Chain Monte Carlo (MCMC) algorithm used in this article. For further details, we refer to the literature. ${ }^{6,10,14}$

Consider the linear model with zero mean additive Gaussian noise e,

$$
\mathbf{b}=A \mathbf{u}+\mathbf{e}, \quad \mathbf{e} \sim \mathscr{N}(0, \Sigma),
$$

where $\Sigma$ is the covariance matrix of e. In addition, the vector $\mathbf{u}$ is assumed to satisfy the a priori bound constraint

$$
C \mathbf{u} \geq \mathbf{c} .
$$

The model (12) defines the likelihood,

$$
\pi(\mathbf{b} \mid \mathbf{u}) \propto \exp \left(-\frac{1}{2}(A \mathbf{u}-\mathbf{b})^{\mathrm{T}} \Sigma^{-1}(A \mathbf{u}-\mathbf{b})\right) .
$$




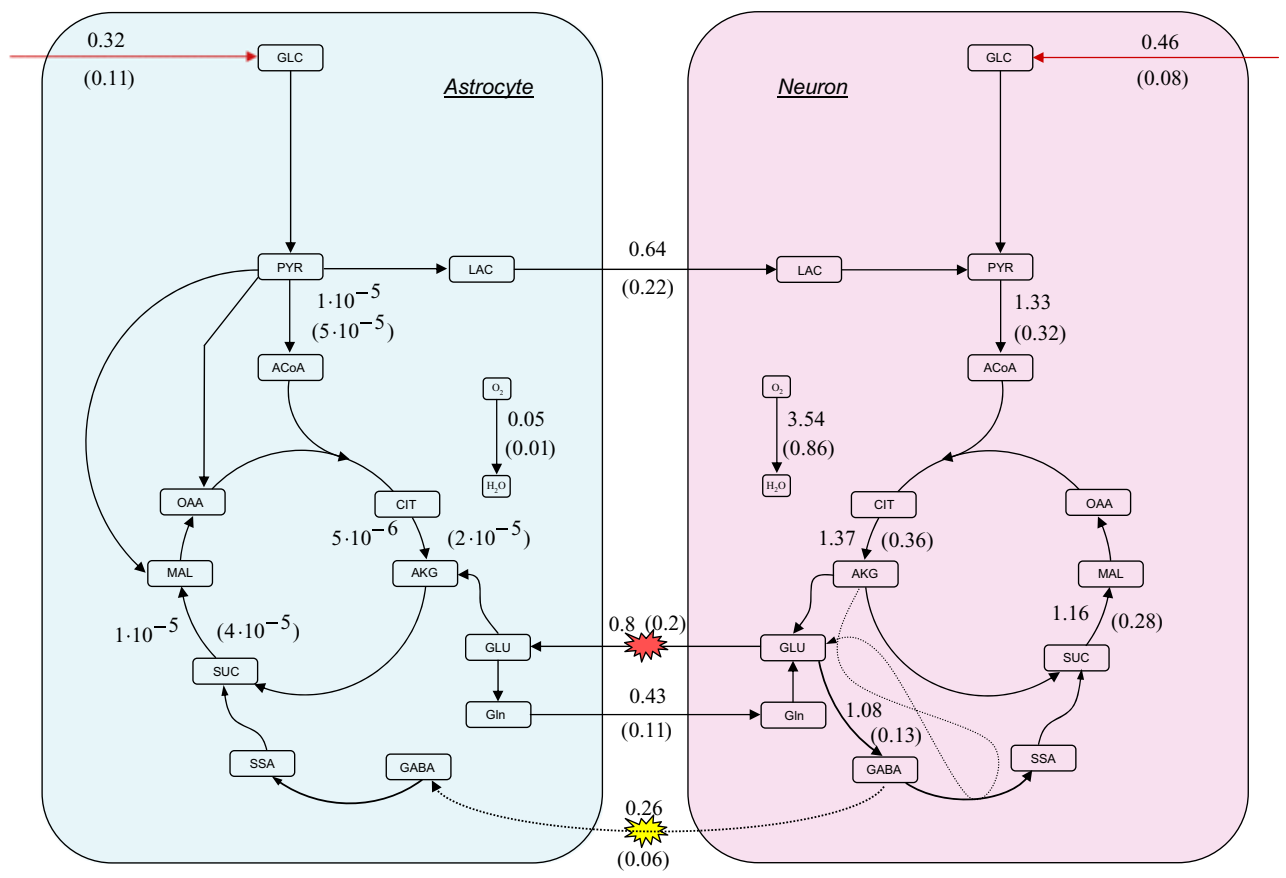

FIGURE 6. The pathway diagrams indicate the activity levels of the principal biochemical reactions under the assumption that astrocyte and neuron have equal access to glucose supply. The key flux values corresponding to high neural activity are outside parentheses, while the values inside the parentheses correspond to low neural activity. The reported flux values are the posterior conditional means estimated from a sample of size 200,000 . The fluxes are scaled to unit volume and expressed in $\mathrm{mmol} / \mathrm{min}$. For the conversion to commonly used units $\mu \mathrm{mol} / \mathrm{min} / \mathrm{g}$ wet weight, we assume that the volume of brain is about $1.5 \mathrm{~L}$, $45 \%$ of which is occupied by neurons and $25 \%$ by astrocytes, which correspond to 0.68 and $0.38 \mathrm{~L}$, respectively. Assuming that $1 \mathrm{~L}$ corresponds to $1,000 \mathrm{~g}$, we have that $1 \mathrm{mmol} / \mathrm{min}-1.48 \mu \mathrm{mol} / \mathrm{min} / \mathrm{g}$ for neuron, and $1 \mathrm{mmol} / \mathrm{min}-2.67 \mu \mathrm{mol} / \mathrm{min} / \mathrm{g}$ for astrocyte. These conversion rules ignore the volume fractions of subcompartmentalizations into cytosol and mitochondria.

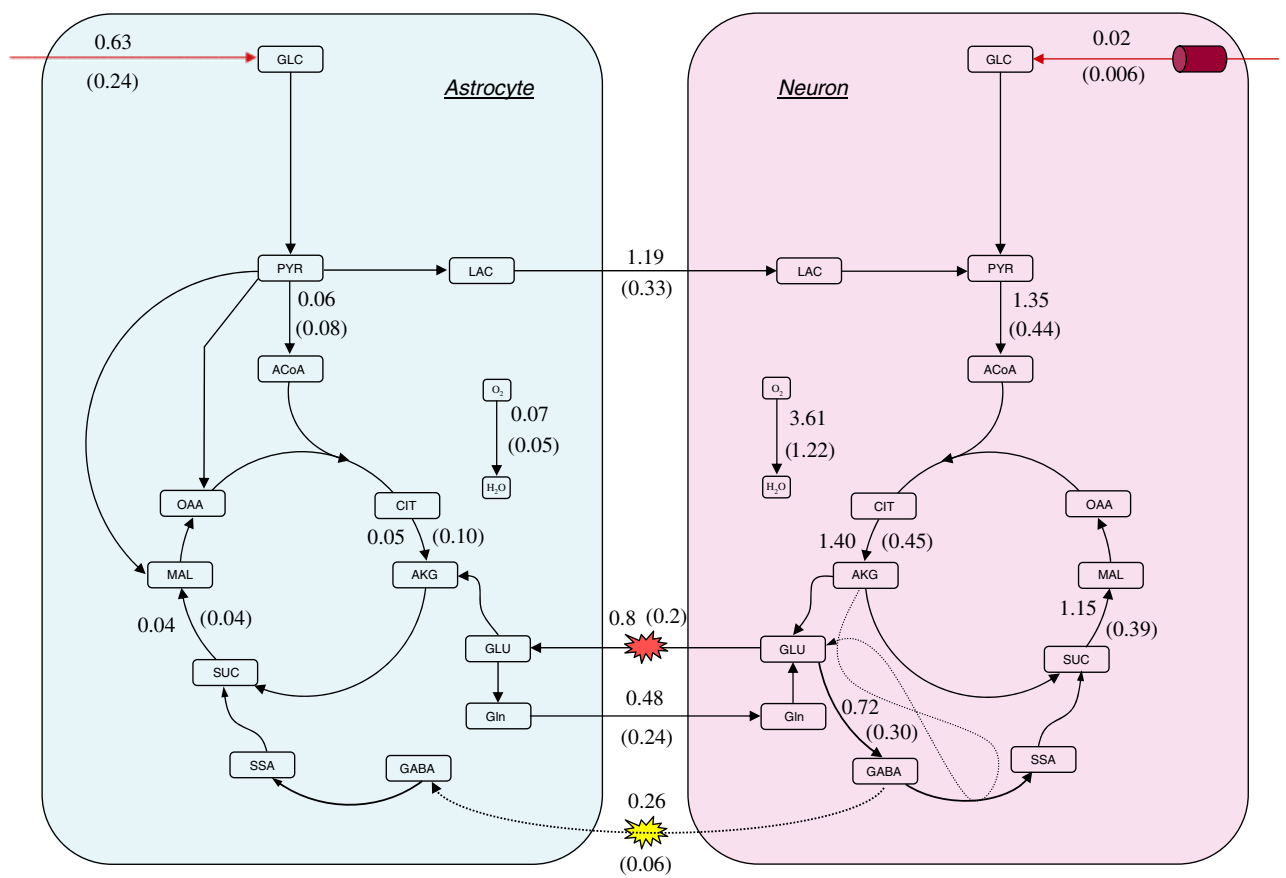

FIGURE 7. The pathway diagrams indicating the activity levels of the principal biochemical reactions under the assumption that neuron has access to less than half of the glucose supply than astrocyte. The key flux values corresponding to high neural activity are outside parentheses, while the values inside the parentheses correspond to low neural activity. The fluxes values are the posterior conditional means and their units are in $\mathrm{mmol} / \mathrm{min}$ as in Fig. 6. 
TABLE 2. Posterior mean values \pm standard deviations (STD) predicted by our model for the most relevant biochemical reactions and transport fluxes for different neural activation levels when we regulate the glucose influx.

\begin{tabular}{|c|c|c|c|c|}
\hline \multirow[b]{3}{*}{ Activity } & \multicolumn{4}{|c|}{ Regulated glucose influx } \\
\hline & \multicolumn{2}{|c|}{0.2} & \multicolumn{2}{|c|}{0.8} \\
\hline & $A$ & $\mathrm{~N}$ & $A$ & $\mathrm{~N}$ \\
\hline$J_{b \rightarrow c, \mathrm{GLC}}$ & $(2.49 \pm 1.45) \times 10^{-1}$ & $(6.21 \pm 5.52) \times 10^{-3}$ & $(6.33 \pm 1.88) \times 10^{-1}$ & $(1.63 \pm 1.06) \times 10^{-2}$ \\
\hline$\Phi_{\mathrm{PYR} \rightarrow \mathrm{AC} O \mathrm{~A}}$ & $(8.66 \pm 6.31) \times 10^{-2}$ & $(4.39 \pm 1.32) \times 10^{-1}$ & $(6.58 \pm 5.13) \times 10^{-2}$ & $1.36 \pm 0.11$ \\
\hline$\Phi_{\mathrm{CIT} \rightarrow \mathrm{AKG}}$ & $(1.02 \pm 6.80) \times 10^{-2}$ & $(4.51 \pm 1.45) \times 10^{-1}$ & $(5.37 \pm 4.44) \times 10^{-2}$ & $1.41 \pm 0.12$ \\
\hline$\Phi_{\mathrm{SUC} \rightarrow \mathrm{MAL}}$ & $(4.59 \pm 3.82) \times 10^{-2}$ & $(3.91 \pm 1.20) \times 10^{-1}$ & $(4.08 \pm 3.50) \times 10^{-2}$ & $1.18 \pm 0.10$ \\
\hline$\Phi_{\mathrm{GLU} \rightarrow \mathrm{GABA}}$ & & $(3.08 \pm 1.50) \times 10^{-1}$ & & $(7.25 \pm 3.04) \times 10^{-1}$ \\
\hline$\Phi_{\mathrm{O}_{2} \rightarrow \mathrm{H}_{2} \mathrm{O}}$ & $(4.89 \pm 1.96) \times 10^{-2}$ & $1.23 \pm 0.22$ & $(7.18 \pm 1.81) \times 10^{-2}$ & $3.61 \pm 0.11$ \\
\hline$J_{A \rightarrow N, \text { LAC }}$ & \multicolumn{2}{|c|}{$(3.33 \pm 1.76) \times 10^{-1}$} & \multicolumn{2}{|c|}{$1.19 \pm 0.22$} \\
\hline$J_{N \rightarrow A, G L U}$ & \multirow{2}{*}{\multicolumn{2}{|c|}{$\frac{0.2}{(243+107) \times 10^{-1}}$}} & \multicolumn{2}{|c|}{0.8} \\
\hline$J_{A \rightarrow N, G \mid n}$ & & & \multicolumn{2}{|c|}{$(4.84 \pm 1.14) \times 10^{-1}$} \\
\hline$J_{N \rightarrow A, G A B A}$ & \multicolumn{2}{|c|}{0.06} & \multicolumn{2}{|c|}{0.24} \\
\hline
\end{tabular}

The larger values of STD are due to the increased $\sigma=0.1$. The value for $J_{b \rightarrow c, \text { GLC }}$ is a net transport and the value for $\Phi_{\text {SUC } \rightarrow \text { MAL }}$ is the average of the two metabolic fluxes $\Phi_{\mathrm{SUC} \rightarrow \mathrm{FUM}}$ and $\Phi_{\mathrm{FUM} \rightarrow \mathrm{MAL}}$.

TABLE 3. Metabolic reaction fluxes in astrocyte.

Cytosol reactions
1. GLC+ATP $\longrightarrow$ G6P+ADP
2. G6P+ATP $\longrightarrow$ GLY+ADP+2Pi
3. GLY+Pi $\longrightarrow$ G6P
4. G6P+ATP $\longrightarrow 2 G A 3 P+A D P$
5. GA3P+Pi+NAD $\longrightarrow$ BPG+NADH
6. BPG+2ADP $\longrightarrow$ PYR+2ATP
7. PYR+NADH $\longrightarrow$ LAC+NAD
8. LAC+NAD $\longrightarrow$ PYR+NADH
9. PCR+ADP $\longrightarrow$ CR+ATP
10. $C R+A T P \longrightarrow P C R+A D P$
11. ADP+Pi $\longrightarrow A T P$
12. ATP $\longrightarrow A D P+P i$

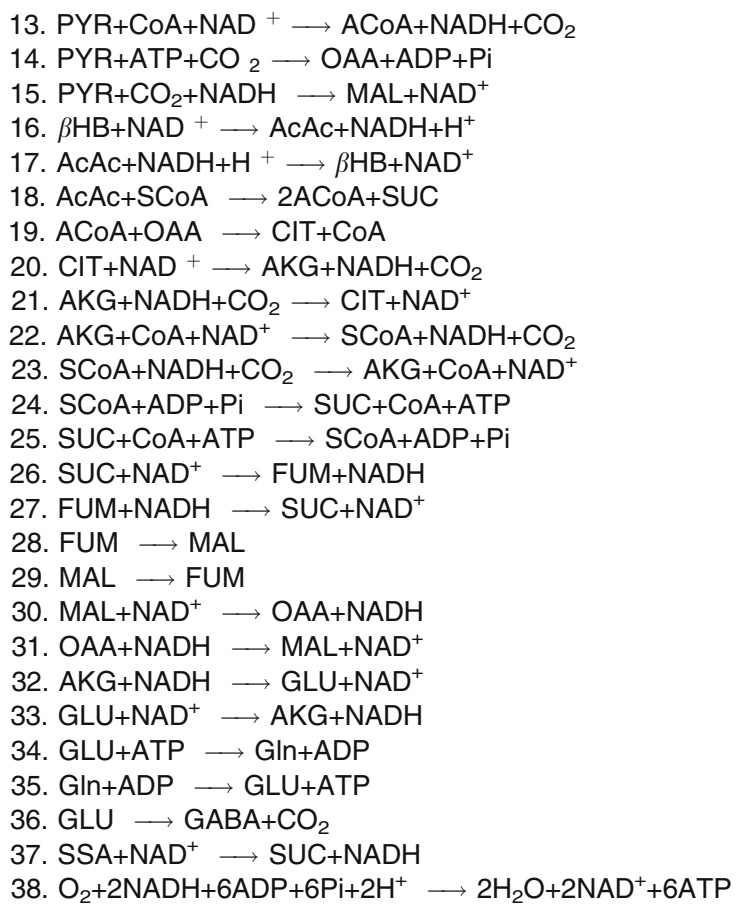

In the present work, the covariance matrix is assumed to be diagonal, the diagonal elements depending on the left hand side of Eq. (12),

$$
\Sigma_{j j}=\max \left(\sigma^{2}, n^{2} b_{j}^{2}\right) .
$$

This choice of $\Sigma$ means that $\sigma^{2}$ expresses our confidence on the validity of the steady state condition in the cell domain, while $n^{2}$ is a measure of the relative uncertainty of the observed arterial blood value $b_{j}$ in the blood domain. By performing a diagonal scaling of the arrays $A$ and $\mathbf{b}$ and redefining $A \rightarrow \Sigma^{-1 / 2} A$ and $\mathbf{b} \rightarrow \Sigma^{-1 / 2} \mathbf{b}$, the likelihood can be written in the more convenient form,

$$
\pi(\mathbf{b} \mid \mathbf{u}) \propto \exp \left(-\frac{1}{2}\|A \mathbf{u}-\mathbf{b}\|^{2}\right) .
$$


TABLE 4. Metabolic reaction fluxes in neuron.

\begin{tabular}{|c|c|}
\hline Cytosol reactions & Mitochondria reactions \\
\hline $\begin{array}{l}\text { 39. } \mathrm{GLC}+\mathrm{ATP} \longrightarrow \mathrm{G} 6 \mathrm{P}+\mathrm{ADP} \\
\text { 40. } \mathrm{G} 6 \mathrm{P}+\mathrm{ATP} \longrightarrow \mathrm{GLY}+\mathrm{ADP}+2 \mathrm{Pi} \\
\text { 41. } \mathrm{GLY}+\mathrm{Pi} \longrightarrow \mathrm{G} 6 \mathrm{P} \\
\text { 42. } \mathrm{G} 6 \mathrm{P}+\mathrm{ATP} \longrightarrow 2 \mathrm{GA3P}+\mathrm{ADP} \\
\text { 43. } \mathrm{GA3P}+\mathrm{Pi}+\mathrm{NAD}+\mathrm{BPG}+\mathrm{NADH} \\
\text { 44. } \mathrm{BPG}+2 \mathrm{ADP} \longrightarrow \mathrm{PYR}+2 \mathrm{ATP} \\
\text { 45. } \mathrm{LAC}+\mathrm{NAD} \longrightarrow \mathrm{PYR}+\mathrm{NADH} \\
\text { 46. } \mathrm{PCR}+\mathrm{ADP} \longrightarrow \mathrm{CR}+\mathrm{ATP} \\
\text { 47. } \mathrm{CR}+\mathrm{ATP} \longrightarrow \mathrm{PCR}+\mathrm{ADP} \\
\text { 48. } \mathrm{ADP}+\mathrm{Pi} \longrightarrow \mathrm{ATP} \\
\text { 49. } \mathrm{ATP} \longrightarrow \mathrm{ADP}+\mathrm{Pi}\end{array}$ & 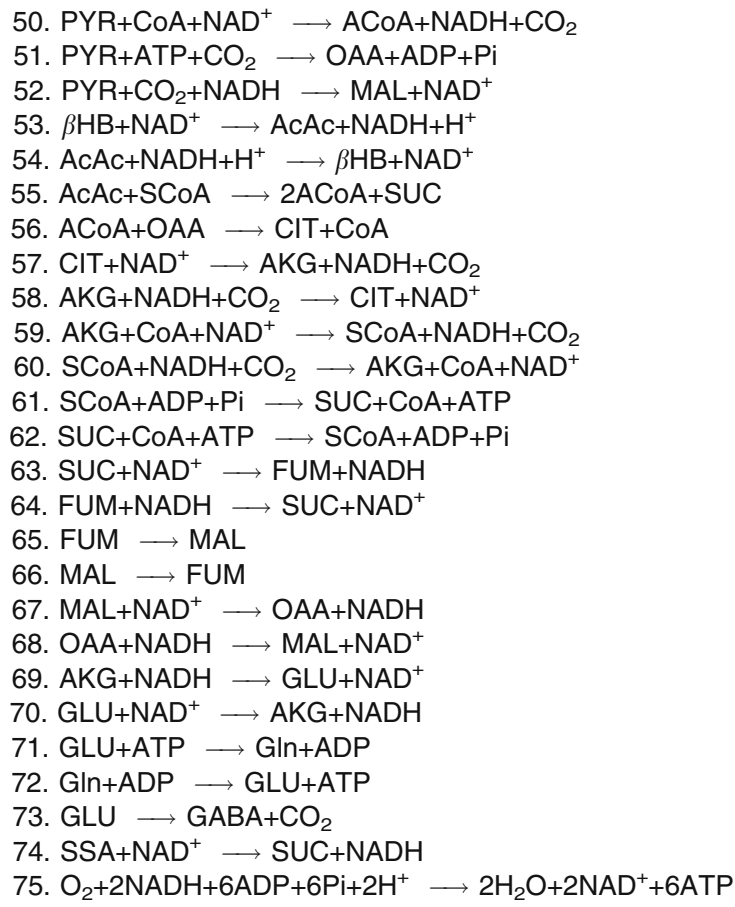 \\
\hline
\end{tabular}

TABLE 5. Metabolic reaction rates in cytosol.

\begin{tabular}{lll}
\hline & \multicolumn{1}{c}{ Astrocyte } & \multicolumn{1}{c}{ Neuron } \\
\hline$R_{\mathrm{GLC}}^{c}$ & $-\Phi_{1}$ & $-\Phi_{39}$ \\
$R_{\mathrm{G} G \mathrm{P}}^{c}$ & $\Phi_{1}+\Phi_{3}-\Phi_{2}-\Phi_{4}$ & $\Phi_{39}+\Phi_{41}-\Phi_{40}-\Phi_{42}$ \\
$R_{\mathrm{GLY}}^{c}$ & $\Phi_{2}-\Phi_{3}$ & $\Phi_{40}-\Phi_{41}$ \\
$R_{\mathrm{GA3P}}^{c}$ & $2 \Phi_{4}-\Phi_{5}$ & $2 \Phi_{42}-\Phi_{43}$ \\
$R_{\mathrm{GPG}}^{c}$ & $\Phi_{5}-\Phi_{6}$ & $\Phi_{43}-\Phi_{44}$ \\
$R_{\mathrm{PYR}}^{C}$ & $\Phi_{6}-\Phi_{7}+\Phi_{8}$ & $\Phi_{44}+\Phi_{45}$ \\
$R_{\mathrm{LAC}}^{C}$ & $\Phi_{7}-\Phi_{8}$ & $-\Phi_{45}$ \\
$R_{\mathrm{P} \mathrm{CR}}^{c}$ & $\Phi_{10}-\Phi_{9}$ & $\Phi_{47}-\Phi_{46}$ \\
$R_{\mathrm{CR}}^{C}$ & $\Phi_{9}-\Phi_{10}$ & $\Phi_{46}-\Phi_{47}$ \\
$R_{\mathrm{ATP}}^{c}$ & $-\Phi_{1}-\Phi_{2}-\Phi_{4}+2 \Phi_{6}-\Phi_{10}+\Phi_{9}-\Phi_{12}+\Phi_{11}$ & $-\Phi_{39}-\Phi_{40}-\Phi_{42}+2 \Phi_{44}-\Phi_{47}+\Phi_{46}-\Phi_{49}+\Phi_{48}$ \\
$R_{\mathrm{ADP}}^{c}$ & $\Phi_{1}+\Phi_{2}+\Phi_{4}-2 \Phi_{6}+\Phi_{10}-\Phi_{9}+\Phi_{12}-\Phi_{11}$ & $\Phi_{39}+\Phi_{40}+\Phi_{42}-2 \Phi_{44}+\Phi_{47}-\Phi_{46}+\Phi_{49}-\Phi_{48}$ \\
$R_{\mathrm{NAD}}^{c}$ & $-\Phi_{5}+\Phi_{7}-\Phi_{8}$ & $-\Phi_{43}-\Phi_{45}$ \\
$R_{\mathrm{NADH}}^{c}$ & $\Phi_{5}-\Phi_{7}+\Phi_{8}$ & $\Phi_{43}+\Phi_{45}$ \\
$R_{\mathrm{Pi}}^{c}$ & $2 \Phi_{2}-\Phi_{3}-\Phi_{5}-\Phi_{11}+\Phi_{12}$ & $2 \Phi_{40}-\Phi_{41}-\Phi_{43}-\Phi_{48}+\Phi_{49}$ \\
\hline
\end{tabular}

In the Bayesian setting, the a priori bound constraints (13) constitute a prior probability density,

$$
\pi_{\text {prior }}(\mathbf{u}) \propto \Theta(C \mathbf{u}-\mathbf{c}),
$$

where $\Theta$ stands for a vectorial Heaviside function, taking on the value one if all the components are nonnegative and vanishing otherwise. The posterior probability density, by Bayes' formula, is proportional to the joint probability density,

$$
\pi(\mathbf{u} \mid \mathbf{b}) \propto \pi_{\text {prior }}(\mathbf{u}) \pi(\mathbf{b} \mid \mathbf{u}) .
$$

The MCMC methods are tools to produce a large ensemble of randomly drawn vectors $\mathbf{u}_{\mathbf{k}}, \quad 1 \leq k \leq N$, that are distributed according to the posterior probability density. The properties of the posterior density can then be estimated from this sample. MCMC methods generate the sample sequentially: the next element in the chain, $\mathbf{u}_{k+1}$, is obtained from $\mathbf{u}_{k}$ by a 
TABLE 6. Metabolic reaction rates in mitochondria.

\begin{tabular}{|c|c|c|}
\hline & Astrocyte & Neuron \\
\hline$R_{\mathrm{PYR}}^{m}$ & $-\Phi_{13}-\Phi_{14}-\Phi_{15}$ & $-\Phi_{50}-\Phi_{51}-\Phi_{52}$ \\
\hline$R_{\mathrm{ACoA}}^{m}$ & $\Phi_{13}+2 \Phi_{18}-\Phi_{19}$ & $\Phi_{50}+2 \Phi_{55}-\Phi_{56}$ \\
\hline$R_{\mathrm{AcAc}}^{m}$ & $\Phi_{16}-\Phi_{17}-\Phi_{18}$ & $\Phi_{53}-\Phi_{54}-\Phi_{55}$ \\
\hline$R_{\beta \mathrm{HB}}^{m}$ & $\Phi_{17}-\Phi_{16}$ & $\Phi_{54}-\Phi_{53}$ \\
\hline$R_{\mathrm{CIT}}^{m}$ & $\Phi_{19}-\Phi_{20}+\Phi_{21}$ & $\Phi_{56}-\Phi_{57}+\Phi_{58}$ \\
\hline$R_{\mathrm{AKG}}^{m}$ & $\Phi_{20}-\Phi_{21}+\Phi_{33}-\Phi_{32}+\Phi_{23}-\Phi_{22}-\Phi_{36}$ & $\Phi_{57}-\Phi_{58}+\Phi_{70}-\Phi_{69}+\Phi_{60}-\Phi_{59}-\Phi_{73}$ \\
\hline$R_{\mathrm{SCOA}}^{m}$ & $\Phi_{22}-\Phi_{23}+\Phi_{25}-\Phi_{24}-\Phi_{18}$ & $\Phi_{59}-\Phi_{60}+\Phi_{62}-\Phi_{61}-\Phi_{55}$ \\
\hline$R_{\mathrm{SUC}}^{m}$ & $\Phi_{24}-\Phi_{25}+\Phi_{27}-\Phi_{26}+\Phi_{37}+\Phi_{18}$ & $\Phi_{61}-\Phi_{62}+\Phi_{64}-\Phi_{63}+\Phi_{74}+\Phi_{55}$ \\
\hline$R_{\mathrm{FUM}}{ }^{m}$ & $\Phi_{26}-\Phi_{27}+\Phi_{29}-\Phi_{28}$ & $\Phi_{63}-\Phi_{64}+\Phi_{66}-\Phi_{65}$ \\
\hline$R_{\mathrm{MAL}}^{m}$ & $\Phi_{28}-\Phi_{29}+\Phi_{31}-\Phi_{30}+\Phi_{15}$ & $\Phi_{65}-\Phi_{66}+\Phi_{68}-\Phi_{67}+\Phi_{51}$ \\
\hline$R_{\mathrm{OAA}}^{m}$ & $\Phi_{30}-\Phi_{31}-\Phi_{19}+\Phi_{14}$ & $\Phi_{67}-\Phi_{68}-\Phi_{56}+\Phi_{50}$ \\
\hline$R_{\mathrm{GLU}}^{m}$ & $\Phi_{32}-\Phi_{33}-\Phi_{34}+\Phi_{35}+\Phi_{36}$ & $\Phi_{69}-\Phi_{70}+\Phi_{71}-\Phi_{72}+\Phi_{73}$ \\
\hline$R_{\mathrm{Gln}}^{m}$ & $\Phi_{34}-\Phi_{35}$ & $-\Phi_{71}$ \\
\hline$R_{\mathrm{GABA}}^{m}$ & $-\Phi_{36}$ & $\Phi_{72}-\Phi_{73}$ \\
\hline$R_{S S A}^{m}$ & $\Phi_{36}-\Phi_{37}$ & $\Phi_{73}-\Phi_{74}$ \\
\hline$R_{\mathrm{ATP}}^{m}$ & $-\Phi_{14}+\Phi_{24}-\Phi_{25}-\Phi_{34}+\Phi_{35}+6 \Phi_{38}$ & $-\Phi_{51}+\Phi_{61}-\Phi_{62}+\Phi_{71}+6 \Phi_{75}$ \\
\hline$R_{\mathrm{ADP}}^{m}$ & $\Phi_{14}-\Phi_{24}+\Phi_{25}+\Phi_{34}-\Phi_{35}-6 \Phi_{38}$ & $\Phi_{51}-\Phi_{61}+\Phi_{62}-\Phi_{71}-6 \Phi_{75}$ \\
\hline$R_{\mathrm{NAD}^{+}}^{m}$ & $\begin{array}{l}-\Phi_{13}+\Phi_{15}-\Phi_{16}+\Phi_{17}-\Phi_{20}+\Phi_{21}-\Phi_{22}+\Phi_{23}+ \\
-\Phi_{26}+\Phi_{27}-\Phi_{30}+\Phi_{31}+\Phi_{32}-\Phi_{33}-\Phi_{37}+2 \Phi_{38}\end{array}$ & $\begin{array}{l}-\Phi_{50}+\Phi_{52}-\Phi_{53}+\Phi_{54}-\Phi_{57}+\Phi_{58}-\Phi_{59}+\Phi_{60}+ \\
-\Phi_{63}+\Phi_{64}-\Phi_{67}+\Phi_{68}+\Phi_{69}-\Phi_{70}-\Phi_{74}+2 \Phi_{75}\end{array}$ \\
\hline$R_{\mathrm{NADH}}^{m}$ & $\begin{array}{l}\Phi_{13}-\Phi_{15}+\Phi_{16}-\Phi_{17}+\Phi_{20}-\Phi_{21}+\Phi_{22}-\Phi_{23}+ \\
+\Phi_{26}-\Phi_{27}+\Phi_{30}-\Phi_{31}-\Phi_{32}+\Phi_{33}+\Phi_{37}-2 \Phi_{38}\end{array}$ & $\begin{array}{l}\Phi_{50}-\Phi_{52}+\Phi_{53}-\Phi_{54}+\Phi_{57}-\Phi_{58}+\Phi_{59}-\Phi_{60}+ \\
\Phi_{63}-\Phi_{64}+\Phi_{67}-\Phi_{68}-\Phi_{69}+\Phi_{70}+\Phi_{74}-2 \Phi_{75}\end{array}$ \\
\hline$R_{\mathrm{CoA}}^{m}$ & $-\Phi_{13}+\Phi_{19}-\Phi_{22}+\Phi_{23}+\Phi_{24}-\Phi_{25}$ & $-\Phi_{50}+\Phi_{56}-\Phi_{59}+\Phi_{60}+\Phi_{61}-\Phi_{62}$ \\
\hline$R_{\mathrm{Pi}}^{m}$ & $\Phi_{14}-\Phi_{24}+\Phi_{25}-6 \Phi_{38}$ & $\Phi_{51}-\Phi_{61}+\Phi_{62}-6 \Phi_{75}$ \\
\hline$R_{\mathrm{O}_{2}}^{m}$ & $-\Phi_{38}$ & $-\Phi_{75}$ \\
\hline$R_{\mathrm{CO}_{2}}^{m}$ & $\Phi_{13}-\Phi_{14}-\Phi_{15}+\Phi_{20}-\Phi_{21}+\Phi_{22}-\Phi_{23}$ & $\Phi_{50}-\Phi_{51}-\Phi_{52}+\Phi_{57}-\Phi_{58}+\Phi_{59}-\Phi_{60}+\Phi_{72}$ \\
\hline
\end{tabular}

TABLE 7. Transport rates.

\begin{tabular}{|c|c|c|}
\hline Astrocyte & Neuron & Between \\
\hline $\begin{array}{l}J_{b \rightarrow c A, \mathrm{GLC}} \\
J_{C A} \rightarrow b, \mathrm{GLC} \\
J_{C A} \rightarrow m A, \mathrm{PYR} \\
J_{b \rightarrow c A, \mathrm{PYR}} \\
J_{C A} \rightarrow b, \mathrm{PYR} \\
J_{b \rightarrow c A, \mathrm{LAC}} \\
J_{C A} \rightarrow b, \mathrm{LAC} \\
J_{m A} \rightarrow c A, \mathrm{ATP} \\
J_{C A} \rightarrow m A, \mathrm{ADP} \\
J_{m A \rightarrow C A, \mathrm{NAD}} \\
J_{C A} \rightarrow m A, \mathrm{NADH} \\
J_{b \rightarrow m A, \beta \mathrm{HB}} \\
J_{m A} \rightarrow b, \beta \mathrm{HB} \\
J_{b \rightarrow m A, \mathrm{O}_{2}} \\
J_{m A \rightarrow b, C O_{2}}\end{array}$ & $\begin{array}{l}J_{b \rightarrow c N, G L C} \\
J_{C N} \rightarrow b, \mathrm{GLC} \\
J_{c N \rightarrow m N, \mathrm{PYR}} \\
J_{b \rightarrow c N, \mathrm{PYR}} \\
J_{C N} \rightarrow b, \mathrm{PYR} \\
J_{b \rightarrow c N, \mathrm{LAC}} \\
J_{C N} \rightarrow b, \mathrm{LAC} \\
J_{m N \rightarrow c N, \mathrm{ATP}} \\
J_{c N \rightarrow m N, \mathrm{ADP}} \\
J_{m N \rightarrow c N, \mathrm{NAD}} \\
J_{C N} \rightarrow m N, \mathrm{NADH} \\
J_{b \rightarrow m N, \beta \mathrm{HB}} \\
J_{m N \rightarrow b, \beta \mathrm{HB}} \\
J_{b \rightarrow m N, \mathrm{O}_{2}} \\
J_{m N \rightarrow b, \mathrm{CO}_{2}}\end{array}$ & $\begin{aligned} & J_{A} \rightarrow N, \text { LAC } \\
& J_{N} \rightarrow A, \text { GLU } \\
& J_{A} \rightarrow N, \text { GIn } \\
& J_{N} \rightarrow A, \text { GABA }\end{aligned}$ \\
\hline
\end{tabular}

The transport between blood and cell domains of oxygen, carbon dioxide, ketone bodies, glutamate and GABA are lumped to direct exchange between blood and mitochondria without passing through the cytosol domain

random move determined by the posterior probability density. The random move strategy is determined by the particular MCMC algorithm that we apply.

In this work Gibbs sampler is the MCMC technique of choice. The random move $\mathbf{u}_{k} \rightarrow \mathbf{u}_{k+1}$ in the Gibbs sampler is done by componentwise random updating, using the conditional densities. More pre- cisely, the updating algorithm can be summarized as follows:

Generate a sample $\left\{\mathbf{u}_{0}, \mathbf{u}_{1}, \ldots, \mathbf{u}_{N}\right\}$, starting from a given initial value $\mathbf{u}_{0}$ satisfying the bound constraints (13), by performing the update $\mathbf{u}_{k} \rightarrow \mathbf{u}_{k+1}$ via the following steps:

(1) with $\mathbf{u}_{-1}=\left(u_{k, 2}, u_{k, 3}, \ldots, u_{k, n}\right)$, draw $u_{k+1,1}$

from $\pi\left(u_{1} \mid \mathbf{u}_{-1}, \mathbf{b}\right)$,

(2) with $\mathbf{u}_{-2}=\left(u_{k+1,1}, u_{k, 3}, \ldots, u_{k, n}\right)$, draw $u_{k+1,2}$

from $\pi\left(u_{2} \mid \mathbf{u}_{-2}, \mathbf{b}\right)$,

(n) with $\mathbf{u}_{-n}=\left(u_{k+1,1}, u_{k+1,2}, \ldots, u_{k+1, n-1}\right)$, draw $u_{k+1, n}$ from $\pi\left(u_{n} \mid \mathbf{u}_{-n}, \mathbf{b}\right)$.

Here we denote by, $\pi\left(u_{j} \mid \mathbf{u}_{-j}, \mathbf{b}\right)$ the conditional density of $u_{j}$ when all the remaining components are fixed to the prescribed value $\mathbf{u}_{-j} \in \mathbb{R}^{n-1}$. To calculate these marginal densities we write the matrix $A$ in terms of its column vectors $\mathbf{a}_{j}$,

$$
A=\left[\begin{array}{llll}
\mathbf{a}_{1} & \mathbf{a}_{2} & \cdots & \mathbf{a}_{n}
\end{array}\right], \quad \mathbf{a}_{j} \in \mathbb{R}^{m},
$$

and letting $A_{-j} \in \mathbb{R}^{m \times(n-1)}$ denote the matrix obtained from $A$ by deleting its $j$ th column, we have

$$
A \mathbf{u}-\mathbf{b}=\mathbf{a}_{j} u_{j}-\mathbf{b}_{j}, \quad \mathbf{b}_{j}=\mathbf{b}-A_{-j} \mathbf{u}_{-j},
$$

and therefore, 


$$
\|A \mathbf{u}-\mathbf{b}\|^{2}=\left\|\mathbf{a}_{j}\right\|^{2} u_{j}^{2}-2 \mathbf{a}_{j}^{\mathrm{T}} \mathbf{b}_{j} u_{j}+\left\|\mathbf{b}_{j}\right\|^{2} .
$$

Therefore, the likelihood function is a Gaussian density proportional to

$$
\exp \left(-\frac{1}{2}\|A \mathbf{u}-\mathbf{b}\|^{2}\right) \propto \exp \left(-\frac{1}{2 \sigma_{j}^{2}}\left(u_{j}-\bar{u}_{j}\right)^{2}\right),
$$

where

$$
\bar{u}_{j}=\frac{\mathbf{a}_{j}^{\mathrm{T}} \mathbf{b}_{j}}{\left\|\mathbf{a}_{j}\right\|^{2}}, \quad \sigma_{j}^{2}=\frac{1}{\left\|\mathbf{a}_{j}\right\|^{2}} .
$$

To find the bounds for $u_{j}$, we write the inequality constraint as

$$
A \mathbf{u}=A_{-j} \mathbf{u}_{-j}+u_{j} \mathbf{a}_{j} \geq \mathbf{c},
$$

and therefore, when $\mathbf{u}_{-j}$ is fixed, the component $u_{j}$ must satisfy the set of inequalities

$$
u_{j} \mathbf{a}_{j} \geq \mathbf{q}_{j}, \quad \mathbf{q}_{j}=\mathbf{c}-A_{-j} \mathbf{u}_{-j} .
$$

To find the (possibly infinite) interval where these inequalities hold, let us assume that, after a possible permutation, the first $\ell$ components of $\mathbf{a}_{j}$ are positive, the remaining negative. Then $u_{j}$ must belong to the interval

$$
u_{j, \min }=\max _{1 \leq i \leq \ell}\left(q_{j, i} / a_{j, i}\right) \leq u_{j} \leq \min _{\ell+1 \leq i \leq m}\left(q_{j, i} / a_{j, i}\right)=u_{j, \max } .
$$

Therefore, the conditional density of $u_{j}$ conditioned on $\mathbf{b}$ and $\mathbf{u}_{-j}$ is

$$
\pi\left(u_{j} \mid \mathbf{u}_{-j}, \mathbf{b}\right) \propto \chi_{j}\left(u_{j}\right) \exp \left(-\frac{1}{2 \sigma_{j}^{2}}\left(u_{j}-\bar{u}_{j}\right)^{2}\right),
$$

where $\chi_{j}$ is the characteristic function of the interval $\left[u_{j, \min }, u_{j, \max }\right]$. The algorithm is therefore reduced to the rather simple task of drawing random numbers from a Gaussian distribution restricted to an interval.

\section{APPENDIX B: BRAIN METABOLISM MODEL}

In this appendix, we tabulate the details concerning the five-compartment metabolic model of the brain.

\section{ACKNOWLEDGMENTS}

This work was supported in part by NIH grant GM-66309 to establish the Center for Modeling Integrated Metabolic Systems at Case Western Reserve University.

\section{REFERENCES}

${ }^{1}$ Aubert, A., and R. Costalat. Interaction between astrocytes and neurons studied using a mathematical model of compartmentalized energy metabolism. J. Cereb. Blood Flow Metab. 25:1476-1490, 2005.

${ }^{2}$ Bonarius, H. P. J., G. Schmid, and J. Tramper. Flux analysis of underdetermined metabolic networks: the quest for the missing constraints. Trends Biotechnol. 15:308-314, 1997.

${ }^{3}$ Calvetti, D., and E. Somersalo. Large scale statistical parameter estimation in complex systems with application to metabolic models. Multiscale Model. Simul. 5(4):13331366, 2006.

${ }^{4}$ Calvetti, D., J. Heino, E. Somersalo, and K. Tunyan. Bayesian stationary state flux balance analysis for a skeletal muscle metabolic model. Inverse Problems and Imaging 1(2):247-263, 2007.

${ }^{5}$ Chih, C.-P., P. Lipton, and E. L. Roberts Jr. Do active cerebral neurons really use lactate rather than glucose? Trends Neurosci. 24(10):573-578, 2001.

${ }^{6}$ Gilks, W. R., S. Richardson, and D. J. Spiegelhalter. Markov Chain Monte Carlo in Practice. London: Chapman \& Hall, 1996.

${ }^{7}$ Gjedde, A., S. Marrett, and M. Vafaee. Oxidative and nonoxidative metabolism of excited neurons and astrocytes. J. Cereb. Blood Flow Metab. 22:1-14, 2002.

${ }^{8}$ Hassel, B. Carboxylation and anaplerosys in neurons and glia. Mol. Neurobiol. 22(1-3):21-40, 2001.

${ }^{9}$ Hyder, F., A. B. Patel, A. Gjedde, D. L. Rothman, K. L. Behar, and R. G. Shulman. Neuronal-glial glucose oxidation and glutamatergic-GABAergic function. J. Cereb. Blood Flow Metab. 26:865-877, 2006.

${ }^{10}$ Kaipio, J., and E. Somersalo. Statistical and Computational Inverse Problems. New York: Springer Verlag, 2004.

${ }^{11}$ Kauffman, K. J., P. Prakash, and J. S. Edwards. Advances in flux balance analysis. Curr. Opin. Biotechnol. 14(5):491496, 2003

${ }^{12}$ LaManna, J. C., and S. Harik. Regional comparisons of brain glucose influx. Brain Res. 326(2):299-305, 1985.

${ }^{13}$ LaManna, J. C., J. F. Harrington, L. M. Vende, K. AbiSalek, W. D. Lust, and S. I. Harik. Regional bood-brain lactate influx. Brain Res. 614(1-2):164-170, 1993.

${ }^{14}$ Liu, J. S. Monte Carlo Strategies in Scientific Computing. New York: Springer Verlag, 2003.

${ }^{15}$ Lowry, O. H., and J. V. Passonneau. The relationships between substrates and enzymes of glycolysis in brain. $J$. Biol. Chem. 239(1):31-42, 1964.

${ }^{16}$ Lowry, O. H., J. V. Passonneau, F. X. Hasselberger, and D. W. Schulz. Effects of ischemia on known substrates and cofactors of the glycolitic pathway in brain. J. Biol. Chem. 239(1):18-30, 1964.

${ }^{17}$ Magistretti, P. J., L. Pellerin, D. L. Rothman, and R. G. Shulman. Energy on demand. Science. 283:496-497, 1999.

${ }^{18}$ Patel, A. B., R. A. de Graaf, G. F. Mason, T. Kanamatsu, D. L. Rothman, R. G. Shulman, and K. L. Behar. Glutamatergic neurotransmission and neuronal glucose oxidation are coupled during intense neuronal activation. J. Cereb. Blood Flow Metab. 24:972-985, 2004.

${ }^{19}$ Patel, A. B., R. A. de Graaf, G. F. Mason, D. L. Rothman, R.G. Shulman, and K. L. Behar. The contribution of GABA to glutamate/glutamine cycling and energy metabolism in the rat cortex in vivo. Proc. Natl. Acad. Sci. U.S.A. 102(15):5588-5593, 2005. 
${ }^{20}$ Pellerin, L., and P. J. Magistretti. Neuroenergetics: calling upon astrocytes to satisfy hungry neurons. Neuroscientist 10(1):53-62, 2004.

${ }^{21}$ Ramakrishna, R., J. S. Edwards, A. McCulloch, and B. O. Palsson. Flux-balance analysis of mitochondrial energy metabolism: consequences of systemic stoichiometric constraints. Am. J. Physiol. Regul. Integr. Comp. Physiol. 280:R695-R704, 2001.

${ }^{22}$ Schilling, C. H., D. Letscher, and B. O. Palsson. Theory for the systemic definition of metabolic pathways and their use in interpreting metabolic function from a pathwayoriented perspective. J. Theor. Biol. 203(3):229-248, 2000.

${ }^{23}$ Schilling, C. H., and B. O. Palsson. The underlying pathway structure of biochemical reaction networks. Proc. Natl. Acad. Sci. U.S.A. 95(8):4193-4198, 1998.

${ }^{24}$ Shulman, R. G., D. L. Rothman, K. L. Behar, and F. Hyder. Energetics basis of brain activity: Implications for neuroimaging. Trends Neurosci. 27(8):489-495, 2004.
${ }^{25}$ Siesjo, B. K. Brain Energy Metabolism. New York: John Wiley \& Sons, 1978.

${ }^{26}$ Varma, A., and B. O. Palsson. Metabolic flux balancing: basic concepts, scientific and practical use. Bio/Technology 12:994-998, 1998.

${ }^{27}$ Williamson, D. H., P. Lund, and H. A. Krebs. The redox state of free nicotinamide-adenine dinucleotide in the cytoplasm and mitochondria of rat liver. Biochem. J. 103(2):514-527, 1967.

${ }^{28}$ Zhou, L., J. E. Salem, G. M. Saidel, W. C. Stanley, and M. E. Cabrera. Mechanistic model of cardiac energy metabolism predicts localization of glycolysis to cytosolic subdomain during ischemia. Am. J. Physiol. Heart Circ. Physiol. 288:2400-2411, 2005. 